\title{
Nonclinical Characterization of the Hypoxia-Inducible Factor Prolyl Hydroxylase Inhibitor Roxadustat, a Novel Treatment of Anemia of Chronic Kidney Disease ${ }^{[\mathbf{S}}$
}

\author{
Ughetta del Balzo, ${ }^{1}$ Pierre E. Signore, ${ }^{1}$ Gail Walkinshaw, Todd W. Seeley, \\ Mitchell C. Brenner, Qingjian Wang, Guangjie Guo, Michael P. Arend, Lee A. Flippin, \\ F. Aisha Chow, David C. Gervasi, Christian H. Kjaergaard, Ingrid Langsetmo, \\ Volkmar Guenzler, David Y. Liu, Steve J. Klaus, Al Lin, and Thomas B. Neff \\ FibroGen, Inc., San Francisco, California
}

Received January 16, 2020; accepted May 13, 2020

\begin{abstract}
Anemia of chronic kidney disease (CKD) is a multifactorial disorder caused by impaired erythropoietin (EPO) production and altered iron homeostasis associated with inflammation. Hypoxia-inducible factor (HIF) is a transcription factor that stimulates erythropoiesis via a coordinated response involving increased EPO production and enhanced iron availability for $\mathrm{Hb}$ synthesis. HIF degradation is regulated by HIF-prolyl hydroxylase (HIF-PH) enzymes. We hypothesized that roxadustat, an orally available small-molecule inhibitor of HIF-PH, would increase EPO production and promote erythropoiesis in animal models of anemia. In cells, roxadustat increased both HIF-1 $\alpha$ and HIF- $2 \alpha$ proteins, leading to an increase in EPO production, even in the presence of EPO-suppressing inflammatory cytokines. Roxadustat administered intermittently to healthy rats and cynomolgus monkeys increased circulating EPO levels, reticulocytes, blood $\mathrm{Hb}$, and hematocrit in a dose-dependent manner. Roxadustat corrected anemia in a rat model of CKD after five-sixth nephrectomy and in a rat model of anemia of inflammation with impaired iron metabolism induced by peptidoglycan-polysaccharide (PG-PS). In the PG-PS model, roxadustat significantly decreased hepatic expression of hepcidin, a hormone responsible for iron sequestration and
\end{abstract}

functional iron deficiency, and increased expression of two genes involved in duodenal iron absorption: divalent metal transporter 1 and duodenal cytochrome b. In conclusion, by activating the HIF pathway, roxadustat increased EPO production, elevated $\mathrm{Hb}$, corrected anemia, and improved iron homeostasis. The coordinated erythropoietic response stimulated by roxadustat, involving both EPO production and mobilization of iron stores, makes this compound a promising treatment of anemia of CKD and anemia associated with functional iron deficiency.

\section{SIGNIFICANCE STATEMENT}

Roxadustat is a novel orally available small-molecule inhibitor of HIF prolyl hydroxylase enzymes that reversibly stabilizes HIF- $\alpha$, thus activating transcription of HIF-dependent genes, including EPO and regulators of iron homeostasis. Activation of the HIF pathway by roxadustat induces erythropoiesis in healthy rats and monkeys and corrects experimentally induced anemia in rats. The coordinated erythropoietic response that increases EPO production and mobilizes iron stores makes roxadustat a promising treatment for anemia of chronic kidney disease and anemia associated with functional iron deficiency.
This research was funded by FibroGen, Inc. U.d.B., P.E.S., G.W., T.W.S., M.C.B., Q.W., G.G., M.P.A., L.A.F., F.A.C., D.C.G., C.H.K., I.L., V.G., D.Y.L. S.J.K., A.L., and T.B.N. are employees, consultants, and/or stock owners of FibroGen, Inc.

${ }^{1}$ U.d.B. and P.E.S. contributed equally to this work.

FibroGen, Inc., San Francisco, CA.

https://doi.org/10.1124/jpet.120.265181.

S This article has supplemental material available at jpet.aspetjournals.org

\section{Introduction}

Anemia is a frequent and serious complication of chronic kidney disease (CKD) that affects millions of patients worldwide (Thomas et al., 2008; Kassebaum et al., 2014; Stauffer and Fan, 2014). Anemia of CKD contributes to decreased quality of life and increased risk of morbidity and mortality (Finkelstein et al., 2009; Thorp et al., 2009). Key factors responsible for anemia of $\mathrm{CKD}$ are a relative deficiency in erythropoietin (EPO) production and a decrease in iron

ABBREVIATIONS: ACD, anemia of chronic disease; BUN, blood urea nitrogen; CKD, chronic kidney disease; DCYTB, duodenal cytochrome b; Dcytb, gene coding for DCYTB protein; DMT1, divalent metal transporter 1; Dmt1, gene coding for DMT1 protein; EGLN egl 9 homolog EPO, erythropoietin; ESA, erythropoiesis-stimulating agent; FIH, factor inhibiting HIF-1 $\alpha$; Hamp, gene coding for hepcidin; Hep3B hepatocellular carcinoma cell lineHIF, hypoxia-inducible factor; HIF-PH, HIF prolyl hydroxylaseHK-2 human proximal tubule kidney cell line; HRP horseradish peroxidaselL, interleukin; $\alpha \mathrm{KG}, \alpha$-ketoglutarate; MCHC, mean corpuscular $\mathrm{Hb}$ concentration; MCV, mean corpuscular volume; MIP, macrophage inflammatory protein; PCR, polymerase chain reaction; PG-PS, peptidoglycan-polysaccharide; PHD, prolyl hydroxylase; S/c11a2, gene coding for DMT1 protein; TNF, tumor necrosis factor; VEGF, vascular endothelial growth factor; WBC, white blood cell. 
availability for $\mathrm{Hb}$ synthesis (Yilmaz et al., 2011; Babitt and Lin, 2012; Locatelli et al., 2017). Currently, anemia of CKD is managed by iron supplementation and, in more severe cases, by administration of supraphysiologic doses of erythropoiesisstimulating agents (ESAs), such as human recombinant EPO, in combination with adjuvant iron therapy (Biggar and Kim, 2017). However, some patients are hyporesponsive to ESAs and need even larger doses because of functional iron deficiency associated with inflammation (MacDougall and Cooper, 2005; Adamson, 2009; Yilmaz et al., 2011). High doses of ESAs increase the risk of serious adverse events, including death, myocardial infarction, congestive heart failure, and stroke (Szczech et al., 2008; Pfeffer et al., 2009). Thus, to effectively treat anemia of $\mathrm{CKD}$, new therapies need to address both impaired EPO production and functional iron deficiency (Locatelli et al., 2017).

The observation that low blood oxygen levels lead to increased EPO production was made decades ago (Prentice and Mirand, 1961; Naets, 1963), but the transcription factors responsible for this hypoxic response were not identified until the 1990s (Semenza and Wang, 1992; Wang and Semenza, 1995; Wang et al., 1995; Tian et al., 1997). Since then, these hypoxia-inducible factors (HIFs) have been found to be part of an oxygen-sensing pathway that is evolutionarily conserved among metazoans (Kaelin, 2005; Kaelin and Ratcliffe, 2008). HIFs function as heterodimers comprising an oxygenregulated $\alpha$-subunit and a constitutively expressed $\beta$-subunit. There are two major HIF- $\alpha$ isoforms, HIF- $1 \alpha$ and HIF- $2 \alpha$, both of which dimerize with the same $\beta$-subunit to form functional HIF-1 and HIF-2 transcription factors, respectively. In the presence of oxygen, HIF- $\alpha$ subunits are hydroxylated on specific proline residues by a family of HIF prolyl hydroxylase (HIF-PH) enzymes comprising three isozymes: PHD1 (Egl nine homolog [EGLN]2), PHD2 (EGLN1), and PHD3 (EGLN3) (Kaelin and Ratcliffe, 2008; Myllyharju, 2013). This hydroxylation enables binding of the von Hippel-Lindau protein, a component of an E3 ubiquitin ligase, which ubiquitinates HIF- $\alpha$ to target it for proteasomal degradation. HIF-PH enzymes require $\alpha$-ketoglutarate $(\alpha \mathrm{KG})$ and oxygen as cosubstrates. Thus, when oxygen levels fall, the activity of HIF-PH enzymes decreases, causing HIF- $\alpha$ subunits to accumulate and dimerize with HIF- $\beta$ to form functional transcription factors. This process induces adaptive changes in gene expression that either enhance oxygen delivery (e.g., erythropoiesis) or promote survival in a hypoxic environment (Kaelin, 2005; Fong and Takeda, 2008; Kaelin and Ratcliffe, 2008). A fourth oxygen and $\alpha$ KG-dependent enzyme, known as factor inhibiting HIF-1 $\alpha$ (FIH), hydroxylates HIF- $\alpha$ subunits on an asparagine residue. Hydroxylation by FIH inhibits binding of transcriptional coactivators to HIF- $\alpha$ and thus limits HIF transcriptional activity (Mahon et al., 2001; Lando et al., 2002a,b).

HIF stimulates erythropoiesis via a coordinated response that involves increases in endogenous EPO and enhanced availability of iron for $\mathrm{Hb}$ synthesis. HIF directly regulates expression of the EPO gene (Haase, 2010; Bunn, 2013), as well as genes encoding proteins involved in iron absorption [e.g., divalent metal transporter 1 (DMT1), duodenal cytochrome b (DCYTB)], iron transport (e.g., transferrin), iron mobilization (e.g., ferroportin), and iron recycling (e.g., heme oxygenase-1) (Haase, 2010). Furthermore, activation of the HIF pathway reduces levels of hepcidin, the hormone responsible for iron homeostasis. Elevated hepcidin, which is driven by chronic inflammation, leads to sequestering of iron in enterocytes, macrophages, and hepatocytes, thereby causing functional iron deficiency. Downregulation of hepcidin by activation of the HIF pathway releases iron trapped in cells, thus increasing iron availability for $\mathrm{Hb}$ synthesis (Peyssonnaux et al., 2008).

Roxadustat was developed as an orally active inhibitor of the HIF-PH enzymes. In this series of in vitro and in vivo studies, we studied the effects of roxadustat on activation of the HIF pathway, EPO secretion, and stimulation of erythropoiesis in normal rats and monkeys. We also evaluated the ability of roxadustat to correct anemia in two different rodent models. The five-sixth nephrectomy model was used to demonstrate that roxadustat can correct anemia of CKD. The model of anemia of chronic disease (ACD) induced by administration of proteoglycan-polysaccharide (PG-PS) was used to show that roxadustat can reverse anemia associated with inflammation and functional iron deficiency.

\section{Materials and Methods}

\section{In Vitro Studies}

HIF Hydroxylase Enzyme Assays. Activity of HIF-PH and FIH enzymes was determined based on the capture of ${ }^{14} \mathrm{CO}_{2}$ released by the decarboxylation of $\left[1{ }^{14} \mathrm{C}\right] \alpha \mathrm{KG}$ (Zhang et al., 1999). Reaction mixtures included $\mathrm{Fe}\left(\mathrm{SO}_{4}\right)$ (Sigma-Aldrich, St. Louis, MO), $\left[1-{ }^{14} \mathrm{C}\right] \alpha$ KG (Perkin Elmer, Waltham, MA), nonlabeled $\alpha$ KG (Sigma-Aldrich), ascorbate (Sigma-Aldrich), peptide substrate (PHD1,2,3: acetylDLDLEMLAPYIPMDDDFQL-amide, FIH: DESGLPQLTSYDCEVNAPIQGSRNLLQGEELLRAL-biotin) (Mimotopes, Mulgrave, Australia), and catalase (catalog number C100; Sigma-Aldrich) in $50 \mathrm{mM}$ HEPES buffer (Corning Life Sciences, Tewksbury, MA), pH 7.4. Enzymatic reactions were initiated by addition of full-length recombinant human HIF-PH or FIH enzyme to the reaction mixture (Hirsilä et al., 2005). The reactions were performed in 96-well microtiter plates (100 $\mu$ l total assay volume) (Thomas Scientific, Santa Clara, CA). ${ }^{14} \mathrm{CO}_{2}$ was captured on a glass fiber filter paper (Inotech Biosystems International, Brandon, FL) soaked with saturated $\mathrm{Ba}(\mathrm{OH})_{2}$ that was laid on top of the 96-well plate. A microtiter plate sealer film (Thermal Seal Insulating Glass, Uxbridge, MA) was applied to the filter paper. The plate and filter paper were sandwiched between two custom-made aluminum plates (Advanced Component Manufacturing, Burlingame, CA) and transferred to a $37^{\circ} \mathrm{C}$ oven for 1 to 2 hours. After incubation, the filter paper was dried in a $103^{\circ} \mathrm{C}$ oven for $40-60$ minutes. To determine percent turnover, aliquots of the reaction mixture were spotted onto the filter paper, and the filter paper was dried again. The dry filter paper was exposed to a storage phosphor screen for 24-72 hours, and the images were recorded with a Typhoon FLA 7000 imager (Amersham Biosciences, Piscataway, NJ). Integrated spot intensities corresponding to control reactions lacking the enzyme were subtracted from integration results for enzyme-containing reactions, and data were converted to enzyme-dependent percent ${ }^{14} \mathrm{CO}_{2}$ release. All enzymatic reactions were run in duplicate.

To determine the $\mathrm{IC}_{50}$ of roxadustat for the HIF-PH and FIH enzymes, a 3-fold dilution series of roxadustat (FG-4592; FibroGen, Inc.) was prepared and added to the reaction mixture (final $1 \% \mathrm{DMSO}$, $\mathrm{v} / \mathrm{v})$ prior to addition of the enzyme. The final reagent concentrations were as follows: $1 \mu \mathrm{M} \mathrm{Fe}\left(\mathrm{SO}_{4}\right), 3 \mu \mathrm{M}\left[1-{ }^{14} \mathrm{C}\right] \alpha \mathrm{KG}, 97 \mu \mathrm{M}$ nonlabeled $\alpha \mathrm{KG}, 1 \mathrm{mM}$ ascorbate, $50 \mu \mathrm{M}$ peptide substrate, and $\sim 2 \mathrm{U} / \mu \mathrm{l}$ catalase. $\mathrm{IC}_{50}$ values were determined by nonlinear fitting using GraFit version 7.0 (Erithacus Software, UK).

To calculate the kinetic parameters for PHD2, turnover velocity was measured as a function of $\alpha \mathrm{KG}$ concentration at varying concentrations of roxadustat. Nonlinear regression analysis was applied to the 
Michaelis-Menten equation to obtain apparent $V_{\max }$ and $K_{\mathrm{m}}$ using GraFit version 7.0. Double-reciprocal plots of the nonlinear best fits at varying roxadustat concentrations were used to evaluate the mode of inhibition, whereas linear fitting of the apparent $K_{\mathrm{m}} / V_{\max }$ versus roxadustat concentration was used to determine $K_{\mathrm{i}}$.

Cell Culture. Hep3B cells (catalog number HB-8064; American Type Culture Collection, Manassas, VA) were maintained in Dulbecco's modified Eagle's medium (catalog number 10-013-CM; Corning Life Sciences) containing 10\% Hyclone FBS (catalog number SH30071.03; GE Healthcare Life Sciences, Pittsburgh, PA) under $10 \% \mathrm{CO}_{2}$ conditions at $37^{\circ} \mathrm{C}$. Treatment medium for Hep3B cells was Dulbecco's modified Eagle's medium containing 0.5\% FBS (D0.5). HK2 cells (catalog number CRL-2190; American Type Culture Collection) were maintained in Keratinocyte Serum-Free Medium supplemented with epidermal growth factor and bovine pituitary extract (catalog number 17005042; Thermo Fisher Scientific, Waltham, MA) under $5 \% \mathrm{CO}_{2}$ conditions at $37^{\circ} \mathrm{C}$. Treatment medium for $\mathrm{HK}-2$ cells was the same as maintenance medium.

Analysis of HIF-1 $\alpha$ and HIF-2 $\alpha$ Stabilization in Hep3B and HK-2 Cells. Hep3B cells and HK-2 cells were plated into $10-\mathrm{cm}$ dishes at $2 \times 10^{6}$ cells or $8 \times 10^{5}$ cells per dish, respectively. The next day, the cells were treated with $3-30 \mu \mathrm{M}$ roxadustat or vehicle control $(0.15 \% \mathrm{DMSO})$ in their respective treatment media. After 3 hours, the cell monolayer was lysed with radio immunoprecipitation assay lysis buffer (Teknova, Hollister, CA) supplemented with Halt protease inhibitors (Thermo Fisher Scientific), and whole-cell lysates were collected.

Analysis of HIF-1 $\alpha$ Protein Levels in Hep3B Cells After Removal of Roxadustat. Hep3B cells were plated into 6-cm dishes at $1 \times 10^{6}$ cells per well. The next day, cells were treated with $30 \mu \mathrm{M}$ roxadustat or vehicle control $(0.15 \% \mathrm{DMSO})$ in treatment medium. After 3 hours, the medium was removed, cells were washed, and fresh D0.5 medium (without roxadustat or vehicle control) was added. Whole-cell lysates, generated in radio immunoprecipitation assay lysis buffer, were collected immediately (time "0"), 15, 30, or 60 minutes after removal of roxadustat.

Western Blot Analyses. Protein concentrations of whole-cell lysates were measured using the Pierce BCA protein assay kit (Thermo Fisher Scientific). Equal protein was loaded for each sample onto electrophoresis gels. The samples were resolved by SDS-PAGE, transferred to a polyvinylidene difluoride membrane (Invitrogen, Carlsbad, CA), and immunoblotted with HIF-1 $\alpha$ (1:1000 dilution, catalog number 610959, lot number 23681; BD Biosciences, San Jose, CA) or HIF-2 $\alpha$ (1:1000 dilution, catalog number AF2886, lot number VEA0312021; R\&D Systems, Minneapolis, MN) antibodies. The horseradish peroxidase (HRP)-conjugated secondary antibodies used were anti-mouse HRP (1:5000 dilution, catalog number 616520; Invitrogen) and anti-goat HRP (1:5000 dilution, catalog number 611620; Invitrogen), respectively. Bands were visualized using SuperSignal West Pico Chemiluminescent Substrate (Thermo Fisher Scientific) according to the manufacturer's instructions. For analysis of the tubulin loading control, polyvinylidene difluoride membranes were stripped with Blot Restore Membrane Rejuvenation Kit (MilliporeSigma, Burlington, MA) prior to immunoblotting with an antitubulin antibody (1:20,000 dilution, catalog number T6199, lot number 102M4773V; MilliporeSigma) and an anti-mouse HRP secondary antibody (1:20,000 dilution, catalog number 616520; Invitrogen). Scanned Western blots were quantified using ImageJ software (NIH, Bethesda, MD). Images were converted to eight-bit images with minimum-maximum scales of 0-255. Selected bands of interest were quantified (mean gray values). Background corrected values for HIF- $\alpha$ and tubulin were then used to calculate relative levels of HIF- $\alpha$ normalized to tubulin levels (HIF- $\alpha$ /tubulin). For graphical representation, HIF- $\alpha$ /tubulin levels were normalized to the $30 \mu \mathrm{M}$ roxadustat condition for each set of samples. For quantification of HIF- $\alpha$ levels in the washout experiment, HIF- $\alpha$ /tubulin levels were normalized to the $30 \mu \mathrm{M}$ roxadustat, 0 -minute washout sample.
Roxadustat Induction of Secreted EPO in Hep3B Cells. Hep3B cells were plated into 96 -well plates at $2 \times 10^{4}$ cells per well. The next day, cells were treated with $3-60 \mu \mathrm{M}$ roxadustat or vehicle control $(0.15 \% \mathrm{DMSO})$ in treatment medium. After 72 hours, conditioned media were collected for EPO analysis by sandwich immunoassay (catalog number DEP00; R\&D Systems).

Effects of Inflammatory Cytokines on Secreted EPO in the Presence or Absence of Roxadustat in Hep3B Cells. Hep3B cells were plated into 96 -well plates at $5.5 \times 10^{4}$ cells per well. The next day, cells were treated with tumor necrosis factor (TNF) $\alpha$ (catalog number 210-TA; R\&D Systems), interleukin (IL)-1 $\beta$ (catalog number 201-LB; R\&D Systems), or PBS vehicle control plus either $3-30 \mu \mathrm{M}$ roxadustat or $0.15 \%$ DMSO in treatment medium. After 72 hours, conditioned media were collected for EPO analysis by sandwich immunoassay (catalog number K15122B; Meso Scale Diagnostics, Rockville, MD).

\section{In Vivo Studies}

Procedures in Normal Rats and Monkeys. Pharmacokinetic parameters for roxadustat were determined in normal male SpragueDawley rats ( $n=4$ per group) of approximately 8 weeks of age under fasting conditions (Tsukuba Laboratories, Nemoto Science Co., Ibaraki, Japan). All procedures were approved by the Institutional Animal Care and Use Committee of Nemoto Science. Animals were housed individually in metal cages and kept on a 12-hour light/dark cycle (lighting: 7:00 AM to 7:00 PM) in an animal holding room with temperature ranging from 20 to $26^{\circ} \mathrm{C}$ and humidity ranging from $40 \%$ to $70 \%$. Animals weighed $275-311 \mathrm{~g}$ at the time of drug administration. A dose of $20 \mathrm{mg} / \mathrm{kg}$ was administered orally by gavage in a volume of $5 \mathrm{ml} / \mathrm{kg}$ ( $4 \mathrm{mg} / \mathrm{ml}$ suspension in $0.5 \%$ sodium carboxymethylcellulose and $0.1 \%$ Tween 80 ). The same dose was administered intravenously via the tail vein at a volume of $0.5 \mathrm{ml} / \mathrm{kg}(40 \mathrm{mg} / \mathrm{ml}$ in a saline solution with $\mathrm{pH}$ adjusted by $\mathrm{NaOH}$ for solubility). Blood samples were collected at times ranging from 0.1 to 48 hours postdose for determination of plasma roxadustat concentration by liquid chromatography-mass spectrometry.

The time course of EPO secretion in plasma after roxadustat administration was determined in male Sprague-Dawley rats $(n=3)$. Procedures were carried out at FibroGen, Inc. (San Francisco, CA) and approved by the Institutional Animal Care and Use Committee at FibroGen, Inc. Animals were housed individually in standard shoebox-style rat cages. Fluorescent lighting was provided for 12 hours per day between 7:00 AM and 7:00 PM. Temperature was maintained between 20 and $22^{\circ} \mathrm{C}$, and humidity was maintained between $35 \%$ and $50 \%$. Rodent Laboratory Diet (catalog number 5001; PMI Feeds, Inc., St Louis, MO) was withheld overnight before dosing and made available 4 hours postdose. Tap water was available ad libitum. Animals weighed $311-318 \mathrm{~g}$ at the time of dosing. A single dose of $30 \mathrm{mg} / \mathrm{kg}$ was administered orally in a volume of $5 \mathrm{ml} / \mathrm{kg}[6 \mathrm{mg} / \mathrm{ml}$ suspension in $0.5 \%$ sodium carboxymethylcellulose (Spectrum Chemical, Gardena, CA) and $0.1 \%$ polysorbate 80 (Avantor Performance Materials, Radnor, PA)]. Blood samples were collected at regular intervals up to 24 hours postdose for determination of plasma EPO and roxadustat concentrations. Plasma EPO was measured using the Quantikine Mouse Erythropoietin ELISA kit (R\&D Systems).

The erythropoietic effect of roxadustat was investigated in male Sprague-Dawley rats (Crl: CD) purchased from Charles River Laboratories Japan, Inc. (Hino Breeding Center, Japan). All procedures were carried out in the animal facility at Nihon Bioresearch, Inc. (Hashima, Japan) following the Basic Guidelines for the Use of Experimental Animals in Institutions under the jurisdiction of the Japanese Ministry of Health, Labor and Welfare and the Guidelines for Management and Welfare of Experimental Animals (Nihon Bioresearch, Inc.). The animals were kept on a 12-hour light/dark cycle (lighting: 6:00 AM to 6:00 PM) in an animal holding room set at approximately $23^{\circ} \mathrm{C}$ (range: $21.8-24.1^{\circ} \mathrm{C}$ ) and $55 \%$ humidity (range: $44.0 \%-61.1 \%$ ) with filtered fresh air changes of 12 times per hour. The animals were housed individually in stainless-steel suspended cages 
and were given free access to solid feed (CRF-1; Oriental Yeast Co., Ltd., Japan) and tap water. Animals weighed $287-338 \mathrm{~g}$ at study initiation and were assigned to treatment groups such that the mean body weight was similar among groups ( $n=8$ per group). Animals were then treated orally by gavage three times per week for 29 days with vehicle or $6.25,12.5,25$, or $50 \mathrm{mg} / \mathrm{kg}$ roxadustat formulated in $0.5 \%$ sodium carboxymethylcellulose (Maruishi Pharmaceuticals, Japan) and $0.1 \%$ Tween 80 (Sigma-Aldrich Japan, Japan). The day before administration of the first dose, and before dosing on days 4,8 , 15 , and 29 of treatment, about $300 \mu \mathrm{l}$ of venous blood was collected to measure blood $\mathrm{Hb}$ concentration, hematocrit, and reticulocyte number with an XT-2000iV automated hematology analyzer (Sysmex Corporation, Japan). On the last day of treatment, approximately 6 hours after dosing, blood was collected from the abdominal aorta in Venoject II vacuum heparinized tubes (Terumo Corporation, Japan). After centrifugation at $4^{\circ} \mathrm{C}$ and $3000 \mathrm{rpm}$ (about 1972g) for 15 minutes, plasma was collected to measure EPO concentration with a Mouse/Rat Immunoassay (R\&D Systems).

The erythropoietic effect of roxadustat was also investigated in normal cynomolgus monkeys. All procedures were carried out at Covance Laboratories, Inc. (Vienna, VA) in accordance with the US Food and Drug Administration Good Laboratory Practice Regulations, Title 21 of the US Code of Federal Regulations Part 58. Male and female monkeys were housed individually in stainless-steel cages. Animals were offered Certified Primate Diet number 2055C (Harlan Teklad) once or twice daily. Water was provided ad libitum. Environmental controls for the animal room were set to maintain $18-29^{\circ} \mathrm{C}$, a relative humidity of $30 \%-70 \%$, a minimum of 10 room air changes per hour, and a 12-hour light/dark cycle. The light/dark cycle was interrupted on occasion for study-related activities. In total, 25 male and 25 female cynomolgus monkeys weighing $1.9-2.5 \mathrm{~kg}$ were used. Blood samples were collected via venipuncture from the femoral vein of nonsedated conscious animals. The animals were randomly assigned to $0,3,10,20$, or $30 \mathrm{mg} / \mathrm{kg}$ roxadustat, with five animals per sex per group. Animals were orally dosed intermittently (three times per week) for 52 weeks. Blood samples were collected to measure blood $\mathrm{Hb}$ concentration and hematocrit with a Siemens Advia 120 twice prior to the first dose and at regular intervals during the 52 weeks of treatment.

Pharmacokinetic parameters for roxadustat were determined in normal male and female cynomolgus monkeys ( $n=3$ per group per sex) from a non-naïve pharmacokinetic colony using a complete-crossover design under overnight fasting conditions (Covance, Madison, WI). All procedures were in compliance with the Animal Welfare Act regulations (Title 9 of the US Code of Federal Regulations Part 3). Animals were individually housed in stainless-steel cages for the duration of the study. At the time of dosing, body weight ranged from 3.0 to $4.1 \mathrm{~kg}$ for males and from 2.9 to $3.4 \mathrm{~kg}$ for females. A dose of $30 \mathrm{mg} / \mathrm{kg}$ was administered orally by gavage in a volume of $5 \mathrm{ml} / \mathrm{kg}(6 \mathrm{mg} / \mathrm{ml}$ suspension in $0.5 \%$ sodium carboxymethylcellulose and $0.1 \%$ Tween 80 ). The same dose was administered intravenously as a slow bolus infusion over approximately 3 minutes via a saphenous vein at $3 \mathrm{ml} / \mathrm{kg}$ $(10 \mathrm{mg} / \mathrm{ml}$ solution in PBS with $\mathrm{pH}$ adjusted by $\mathrm{NaOH}$ for solubility). Blood samples were collected at regular intervals for up to 96 hours postdose for determination of plasma roxadustat concentration by liquid chromatography-mass spectrometry.

Rat Model of Anemia After Five-Sixth Nephrectomy. Male Sprague-Dawley rats (Charles River Laboratories, Hollister, CA) were used to determine the erythropoietic effects of roxadustat in an anemia model of kidney injury induced by five-sixth nephrectomy. All procedures were performed in the animal facility at FibroGen, Inc. and were approved by the Institutional Animal Care and Use Committee at FibroGen, Inc. Animals were housed in pairs in standard shoebox-style rat cages. Fluorescent lighting was provided for 12 hours per day between 7:00 AM and 7:00 PM. Temperature was maintained between 20 and $22^{\circ} \mathrm{C}$, and humidity was maintained between $40 \%$ and $70 \%$. Rodent Laboratory Diet (catalog number 5001; PMI Feeds, Inc.) and tap water were available ad libitum. Animals weighed approximately $300 \mathrm{~g}$ at study initiation. Rats were anesthetized with isoflurane (Isoflo; Patterson Veterinary, Dinuba, CA), and a midline abdominal incision was made under sterile conditions followed by blunt dissection of the renal pedicles. Two or three branches of the left renal artery were ligated to infarct two-thirds of the kidney, as indicated by a change in kidney color. The right kidney was then removed, and the abdominal incision was closed. Sham animals underwent the same procedure without left renal artery ligation and right nephrectomy. Animals recovered for 5 weeks until anemia and kidney dysfunction developed, as assessed by complete blood cell count (Cell-Dyn 3700; Abbott Diagnostics, Abbott Park, IL) and serum concentrations of blood urea nitrogen (BUN) and creatinine (Quality Clinical Laboratories, Mountain View, CA). Nephrectomized animals were then assigned to treatment with vehicle or roxadustat such that all groups had similar mean baseline body weight, hematocrit, $\mathrm{Hb}$, and serum creatinine. Sham animals were similarly assigned to groups. Nephrectomized animals were treated orally by gavage three times per week for 1 week, followed by twice per week for a $2 \mathrm{nd}$ week with vehicle or with 20 or $40 \mathrm{mg} / \mathrm{kg}$ roxadustat (FibroGen, Inc.) formulated in $0.5 \%$ sodium carboxymethylcellulose (Spectrum Chemical) and $0.1 \%$ polysorbate 80 (Avantor Performance Materials) ( $n=8$ to 9 per group). Sham animals were treated with vehicle or $20 \mathrm{mg} / \mathrm{kg}$ of roxadustat using the same dosing regimen as nephrectomized animals $(n=8$ per group). Venous blood samples were collected after 7 and 14 days of treatment of blood cell count and serum chemistry analysis.

Rat Model of Anemia of Inflammation. Female Lewis rats (Harlan Laboratories, Indianapolis, IN) were used to determine the erythropoietic effect of roxadustat in a model of anemia of inflammation. All procedures were performed in the animal facility at FibroGen, Inc., and were approved by the Institutional Animal Care and Use Committee at FibroGen, Inc. Animals were individually housed in standard shoebox-style rat cages. Fluorescent lighting was provided for 12 hours per day between 7:00 AM and 7:00 PM. Temperature was maintained between 20 and $22^{\circ} \mathrm{C}$, and humidity was maintained between $35 \%$ and 50\%. Purina Rodent Chow (catalog number 5001; PMI Nutrition International, Inc., Brentwood, MO) and tap water were available ad libitum. Animals weighed 153-176 $\mathrm{g}$ at study initiation. PG-PS polymers from Streptococcus pyogenes (PG-PS 10S; Lee Laboratories, Grayson, GA) were administered intraperitoneally in physiologic saline at $15 \mathrm{mg} / \mathrm{kg}$ of rhamnose to induce anemia. Sham (unchallenged) animals were administered physiologic saline. Four weeks after injection of PG-PS, complete blood cell count was determined (CellDyn 3700; Abbott Diagnostics), and animals were assigned to treatment groups such that all PG-PS-challenged groups had similar mean baseline hematocrits. PG-PS-challenged animals with hematocrit above $35 \%$ were considered nonresponsive and were not used in the study. Unchallenged animals were similarly assigned to groups. Animals were then treated orally by gavage three times per week with $30 \mathrm{mg} / \mathrm{kg}$ roxadustat formulated in $0.5 \%$ sodium carboxymethylcellulose (Spectrum Chemical) and $0.1 \%$ polysorbate 80 (Avantor Performance Materials) ( $n=8$ PG-PS-challenged; $n=4$ unchallenged) or vehicle ( $n=8$ PG-PS-challenged; $n=4$ unchallenged). Animals were also treated intravenously with darbepoetin alfa (Aranesp; Amgen, Thousand Oaks, CA) every other week at $30 \mu \mathrm{g} / \mathrm{kg}$ ( $n=7$ PG-PS-challenged; $n=4$ unchallenged) or sodium ferric gluconate complex (Ferrlecit; Watson Pharma) once per week at $1.5 \mathrm{mg} / \mathrm{kg}$ ( $n=7$ PG-PS-challenged; $n=4$ unchallenged). Venous blood samples were collected after 14 and 28 days of treatment for blood cell count and after 28 days for serum iron and cytokine measurements. A multiplex cytokine panel was run for IL- $1 \alpha$, IL- $1 \beta$, IL-4, TNF $\alpha$, granulocyte-macrophage colony-stimulating factor, IL6 , and macrophage inflammatory protein (MIP)- $3 \alpha$ on serum samples. After the last blood sample, animals were euthanized, and their front paws were harvested and weighed to quantify inflammation. Liver and duodenum were harvested and processed for quantitative real-time PCR. 
Gene Expression Analysis. Liver and duodenum tissue samples were stored in Ambion RNAlater solution (Thermo Fisher Scientific). RNA was isolated by homogenization in Trizol (Invitrogen) and further purified on RNeasy96 columns (Qiagen, Germany). Complementary DNA was prepared from total RNA using Omniscript reverse transcriptase (Qiagen) and random hexamer primers. Quantitative real-time PCR was performed in a Prism 7000 (Applied Biosystems, Foster City, CA) instrument using TaqMan Universal PCR Master Mix (Applied Biosystems) and TaqMan Assays-on-Demand (Applied Biosystems) assays. Quantitative PCR data for the duodenal cytochrome b gene (Dcytb), Dmt1 (also known as Slc11a2, iron transporter DMT1 gene), and Hamp (hepcidin gene) were normalized to $18 \mathrm{~S}$ ribosomal RNA expression.

Statistics. All in vitro data are presented as means \pm S.D.. Data from cell-based studies were analyzed using ANOVA followed by Dunnett's multiple comparison test. All in vivo data are presented as means \pm S.E.M.. Data from in vivo studies were analyzed using ANOVA followed by a multiple comparison procedure at each time point (Student-Newman-Keuls method). Steel's test was performed to analyze in vivo EPO results. When values of EPO were below the lower limit of quantification $(47.0 \mathrm{pg} / \mathrm{ml})$, the lower limit of quantification was used for statistical analysis. A $P$ value less than 0.05 was considered statistically significant for all tests.

\section{Results}

Roxadustat is an Inhibitor of HIF-PH Enzymes that Reversibly Stabilizes HIF-1 $\alpha$ and HIF-2 $\alpha$. Roxadustat (FG-4592) was selected from a series of 4-hydroxyisoquinoline glycinamides that were designed to inhibit HIF-PH enzymes by competing with the substrate $\alpha \mathrm{KG}$. The ability of roxadustat to inhibit recombinant full-length human HIF-PH enzymes was measured using an enzyme assay based on the decarboxylation of ${ }^{14} \mathrm{C}$-labeled $\alpha \mathrm{KG}$ and the production of ${ }^{14} \mathrm{CO}_{2}$ (Zhang et al., 1999). In the presence of $100 \mu \mathrm{M} \alpha \mathrm{KG}$, $1 \mu \mathrm{M} \mathrm{Fe}^{2+}$, and $1 \mathrm{mM}$ ascorbate, roxadustat $\mathrm{IC}_{50}$ values of 1.04 $\pm 0.46,1.74 \pm 0.73$, and $0.36 \pm 0.12 \mu \mathrm{M}$ were obtained for PHD1, PHD2, and PHD3, respectively (mean \pm S.D., $n=9$ to 10). The dependence of PHD2 activity on $\alpha \mathrm{KG}$ concentration at several concentrations of roxadustat yielded invariable $V_{\max }$ and variable $K_{\mathrm{m}}$, indicating competitive inhibition kinetics with respect to $\alpha \mathrm{KG}$ and a $K_{\mathrm{i}}$ value for roxadustat of $20 \pm 3 \mathrm{nM}$ (Supplemental Fig. 1; Supplemental Table 1). The selectivity of roxadustat for the HIF-PH enzymes over FIH was determined by measuring roxadustat's ability to inhibit recombinant full-length human FIH under the same assay conditions as those used for the HIF-PH enzymes. Under those conditions, the $\mathrm{FIH} \mathrm{IC} \mathrm{IC}_{50}$ for roxadustat was $>150 \mu \mathrm{M}$.

The ability of roxadustat to stabilize HIF- $\alpha$ was explored in two human cell lines: Hep3B hepatoma cells and HK-2 renal proximal tubule cells. In both lines, incubation with roxadustat for 3 hours dose-dependently increased HIF- $1 \alpha$ and HIF$2 \alpha$ protein levels (Fig. 1A; Supplemental Fig. 2, A and B). The reversibility of HIF- $\alpha$ stabilization by roxadustat was investigated by performing washout studies in Hep3B cells. Compared with DMSO-treated cells, Hep3B cells treated with $30 \mu \mathrm{M}$ roxadustat for 3 hours and then harvested immediately exhibited an accumulation of HIF- $1 \alpha$ protein. In cells treated with roxadustat for 3 hours and harvested at 15,30 , or 60 minutes after washout of the drug, HIF- $1 \alpha$ levels rapidly decreased back to basal levels (Fig. 1B; Supplemental Fig. 2C). A reduction in HIF-1 $\alpha$ protein was observed 15 minutes after washout, and by 60 minutes, HIF- $1 \alpha$ was no longer detectable.
Roxadustat Stimulates EPO Production in Cells and Overcomes the Suppressive Effects of Inflammatory Cytokines. The ability of roxadustat to increase production of EPO, a well known HIF target gene, was investigated in Hep3B cells. Exposure of Hep3B cells to roxadustat dosedependently increased EPO protein secreted into the culture supernatant (Fig. 2A). EPO production is suppressed by proinflammatory cytokines, such as TNF $\alpha$ and IL- $1 \beta$ (Jelkmann et al., 1994; La Ferla et al., 2002). We found that both of these cytokines dose-dependently decreased EPO protein secreted by Hep3B cells (Fig. 2, B and C). We explored whether roxadustat could overcome this suppressed EPO production. Hep3B cells were treated with increasing concentrations of roxadustat in the presence of either $0.125 \mathrm{ng} / \mathrm{ml} \mathrm{TNF} \alpha$ or $0.125 \mathrm{ng} / \mathrm{ml} \mathrm{IL}-1 \beta$, concentrations that robustly suppress EPO production (Fig. 2, B and C). Even in the presence of these proinflammatory cytokines, roxadustat dose-dependently increased EPO production from Hep3B cells (Fig. 2, D and E).

Pharmacokinetics of Roxadustat and Time Course of EPO Secretion in Rats and Monkeys. When dosed intravenously at $20 \mathrm{mg} / \mathrm{kg}$ in rats and $30 \mathrm{mg} / \mathrm{kg}$ in monkeys, roxadustat showed low systemic clearances in rats and monkeys that were much lower than hepatic blood flow, and

A
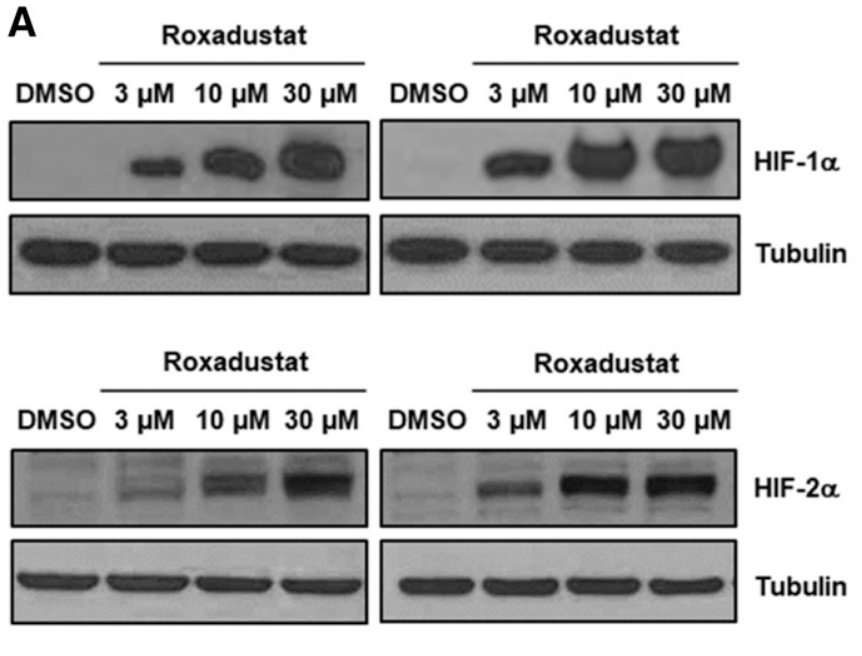

Hep3B Cells

HK-2 Cells

B

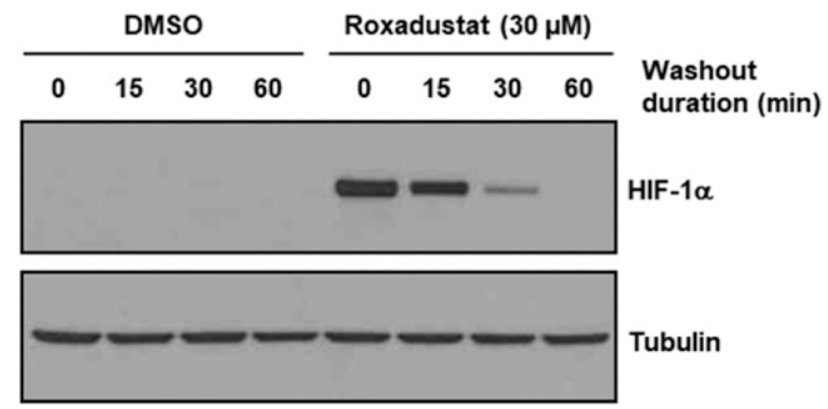

Fig. 1. In vitro accumulation of $\mathrm{HIF}-1 \alpha$ and $\mathrm{HIF}-2 \alpha$ proteins by roxadustat. (A) Western blot of HIF-1 $\alpha$ and HIF- $2 \alpha$ in Hep3B and HK-2 cells 3 hours after exposure to either DMSO or increasing concentrations of roxadustat. (B) Western blot of HIF- $\alpha$ in Hep3B cells treated with DMSO or $30 \mu \mathrm{M}$ roxadustat for 3 hours and then harvested immediately (0 minutes) or after a washout period of 15,30 , or 60 minutes. 


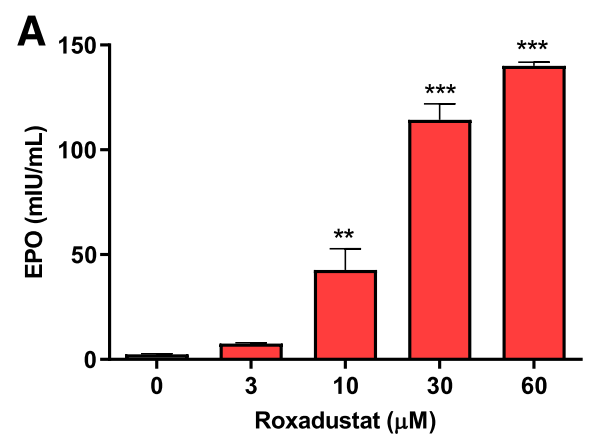

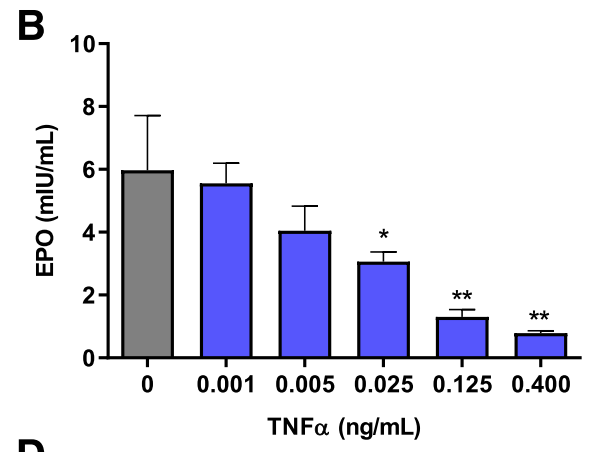

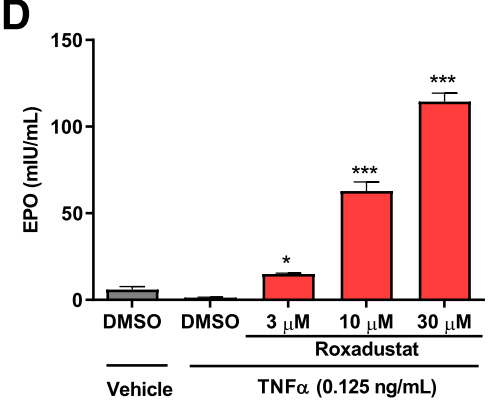

C

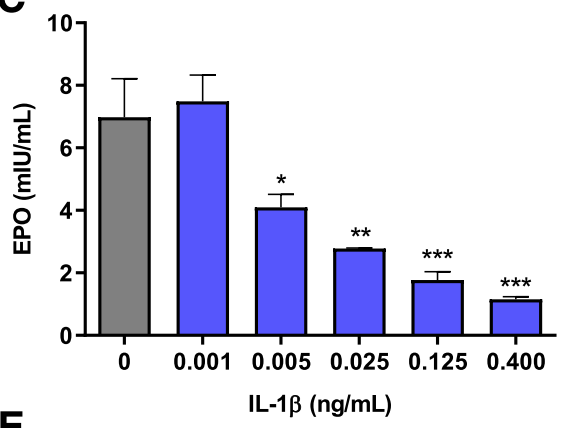

E

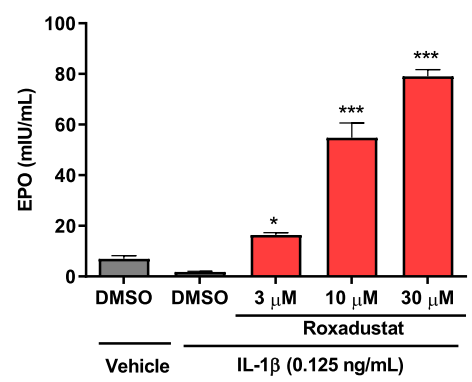

Fig. 2. EPO production in Hep3B cells treated with roxadustat and inflammatory cytokines. (A-C) EPO levels in the media of Hep3B cells treated with increasing concentrations of roxa$\operatorname{dustat}(\mathrm{A}), \mathrm{TNF} \alpha(\mathrm{B})$, or IL-1 $\beta$ (C) for 72 hours. (D and E) EPO levels in the media of Hep3B cells treated with increasing concentrations of roxadustat in the presence of $0.125 \mathrm{ng} / \mathrm{ml} \mathrm{TNF} \alpha$ (D) or $0.125 \mathrm{ng} / \mathrm{ml} \mathrm{IL-1} \beta$ (E). Data are presented as means \pm S.D. $* P<0.05 ; * * P<0.01 ; * * * P<$ 0.001 vs. vehicle group [vs. vehicle plus cytokine group for (D and $\mathrm{E})$ ]. apparent volumes of distribution at steady state (Vdss, 134$178 \mathrm{ml} / \mathrm{kg}$ ) that were larger than plasma volume but less than total body water (Table 1). Oral bioavailability was high in both species $(70 \%-100 \%)$. Plasma roxadustat peaked 2 hours after a $30-\mathrm{mg} / \mathrm{kg}$ oral dose in rats, whereas maximum plasma EPO concentration was achieved 8-12 hours postdose (Fig. 3). Twenty-four hours after dosing, roxadustat plasma concentrations were at low levels (less than $5 \%$ of $C_{\text {max }}$ ), and EPO levels were close to baseline levels.

Roxadustat Increases Circulating EPO Concentration and Erythropoiesis in Normal Rats. The in vivo erythropoietic effect of roxadustat was evaluated in normal rats at doses ranging from 6.25 to $50 \mathrm{mg} / \mathrm{kg}$ that were administered three times per week for 29 days. Blood $\mathrm{Hb}$ concentration and hematocrit gradually increased over time in a dose-dependent manner (Fig 4, A and B). The 12.5-mg/kg dose induced a modest $0.9-\mathrm{g} / \mathrm{dl}$ rise in $\mathrm{Hb}$ over vehicle by day 29 , whereas the high dose $(50 \mathrm{mg} / \mathrm{kg}$ ) increased $\mathrm{Hb}$ by $5.8 \mathrm{~g} / \mathrm{dl}$ compared with vehicle. Reticulocytes also increased in a dosedependent manner, and maximum responses were seen on days 4 and 8 of treatment in the $25-$ and $50-\mathrm{mg} / \mathrm{kg}$ groups, respectively (Fig. 4C). Reticulocytes gradually returned to vehicle levels in the $25-\mathrm{mg} / \mathrm{kg}$ group, but they remained elevated compared with the vehicle group after 29 days of treatment with $50 \mathrm{mg} / \mathrm{kg}$. Plasma EPO concentrations after 29 days of treatment with $12.5,25$, and $50 \mathrm{mg} / \mathrm{kg}$ roxadustat were significantly higher than in the vehicle group (Fig 4D). Plasma EPO was more than seven times higher in the 12.5$\mathrm{mg} / \mathrm{kg}$ group than in the vehicle group, whereas it was more than 300 times higher in the $50-\mathrm{mg} / \mathrm{kg}$ group than in the

TABLE 1

Summary of the pharmacokinetics of roxadustat

Mean \pm S.D. (if available).

\begin{tabular}{|c|c|c|c|c|}
\hline Species & CL (ml/h per kilogram) & Vdss (ml/kg) & $t_{1 / 2}(\mathrm{~h})$ & Oral F (\%) \\
\hline $\operatorname{Rat}^{a}$ & $33.7 \pm 2.7$ & $178 \pm 19$ & $3.6 \pm 1.4$ & 100 \\
\hline Monkey $^{b}$ & $55.7 \pm 12.2$ & $134 \pm 32$ & $10.8 \pm 1.3$ & $70 \pm 17$ \\
\hline
\end{tabular}

${ }^{a}$ Twenty milligrams per kilogram dose, $n=4$ (male) per group, oral $\mathrm{F}$ from non-crossover design.

${ }^{b}$ Thirty milligrams per kilogram dose, $n=6$ (three males and three females) per group, oral F from crossover design. CL, clearance; Oral F, oral bioavailability; Vdss, apparent volume of distribution at steady state. 


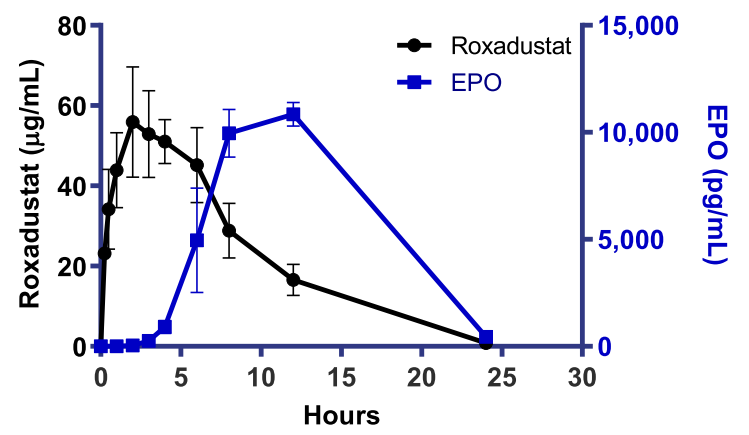

Fig. 3. Plasma roxadustat and EPO concentrations in normal rats after a single $30-\mathrm{mg} / \mathrm{kg}$ oral dose $(n=3)$. Data are presented as means \pm S.E.M.

vehicle group. EPO concentration was below the lower limit of quantification in all vehicle-treated animals and in seven of eight animals in the $6.25-\mathrm{mg} / \mathrm{kg}$ group.

Roxadustat Increases Erythropoiesis in Normal Cynomolgus Monkeys. In a 52-week intermittent-dosing study in male and female cynomolgus monkeys, $\mathrm{Hb}$ was measured prior to the first dose and at regular intervals during the dosing period. Monkeys administered $\geq 20 \mathrm{mg} / \mathrm{kg}$ roxadustat three times per week exhibited increased erythropoiesis, with significant increases in $\mathrm{Hb}$ apparent after 2 weeks of dosing (Fig. 5A). The transient decrease in $\mathrm{Hb}$ observed in all groups during the 1st week of treatment was due to multiple bleeds performed in the days preceding the $\mathrm{Hb}$ measurement to assess drug exposure. Red blood cell count was also significantly elevated at doses $\geq 20 \mathrm{mg} / \mathrm{kg}$ (Fig. 5B).

Roxadustat Corrects Anemia in Rats after Five-Sixth Nephrectomy. The erythropoietic activity of roxadustat was determined in a rat model of anemia of CKD induced by fivesixth nephrectomy. Nephrectomy resulted in anemia, as shown by lower $\mathrm{Hb}$ (Fig. 6A), hematocrit (Fig. 6B), and red blood cell count (Fig. 6C) than sham surgery. Nephrectomized animals also had higher levels of BUN and serum creatinine than sham animals, indicating progressive kidney failure (Supplemental Fig. 3). After 2 weeks of intermittent dosing, treatment with 20 and $40 \mathrm{mg} / \mathrm{kg}$ roxadustat significantly increased and normalized $\mathrm{Hb}$ (Fig. 6A), hematocrit (Fig. 6B), and red blood cell count (Fig. 6C) in nephrectomized animals compared with the vehicle control group. BUN and serum creatinine values were not affected by treatment with roxadustat (Supplemental Fig. 3).

Roxadustat Corrects Anemia of Inflammation in a Rat Model of ACD. The efficacy of roxadustat in correcting anemia was investigated in a rat model of PG-PS-induced chronic inflammation. Hb was below $10 \mathrm{~g} / \mathrm{dl}$ in all PGPS-challenged groups before treatment initiation, indicating severe anemia (Fig. 7A). Mean corpuscular volume (MCV, Fig. 7B) and mean corpuscular $\mathrm{Hb}$ concentration (MCHC, Fig. 7C) were also reduced in anemic animals, indicating microcytosis and hypochromia, respectively.

Treating anemic PG-PS-challenged animals with $30 \mathrm{mg} / \mathrm{kg}$ roxadustat three times per week for 4 weeks increased their $\mathrm{Hb}$ to levels similar to nonchallenged animals administered vehicle (Fig. 7A). Roxadustat also improved the microcytosis (increased MCV, Fig. 7B) and hypochromia (increased MCHC, Fig. 7C) observed in the PG-PS-challenged groups. In contrast, darbepoetin and intravenous iron had no effect on $\mathrm{Hb}$, MCV, or MCHC in anemic PG-PS-challenged animals.

In nonchallenged animals, treatment with either roxadustat or darbepoetin produced a robust erythropoietic response. After 2 weeks of treatment with roxadustat or darbepoetin, $\mathrm{Hb}$ was significantly higher than in the vehicle group, and the erythropoietic response was maintained at 4 weeks with both treatments (Fig. 7A). Intravenous iron had no effect on erythropoiesis in nonchallenged animals (Fig. 7A).

Roxadustat Does Not Affect the Inflammatory Response in a Rat Model of ACD. Animals challenged with PG-PS developed an inflammatory response. These animals exhibited increased circulating white blood cells (WBCs),
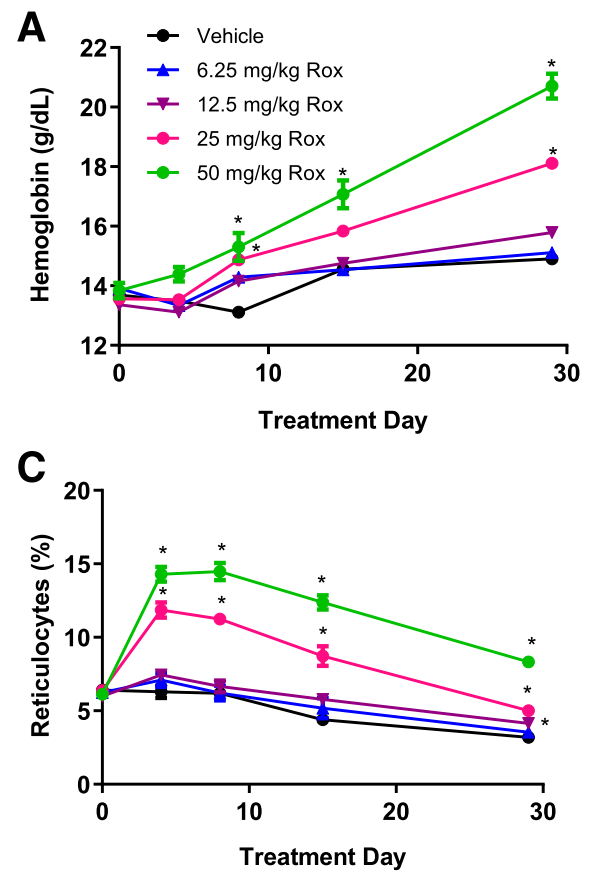

B

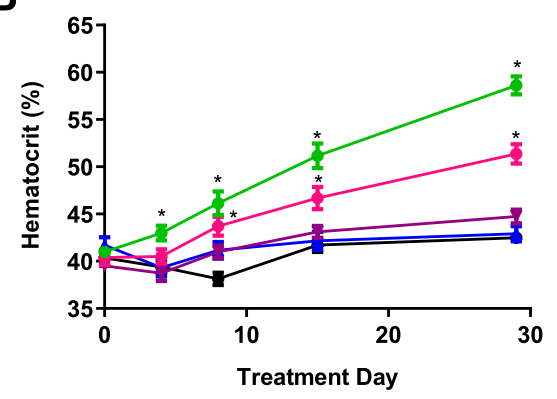

D

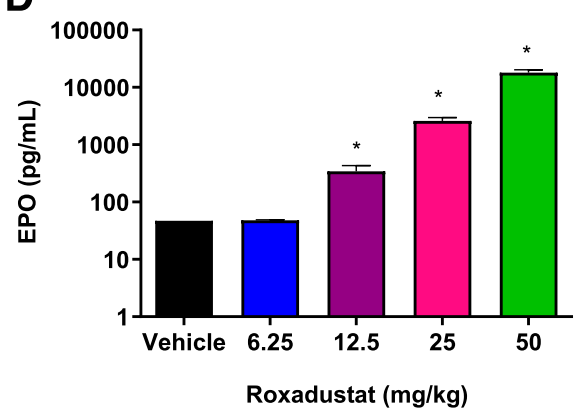

Fig. 4. Blood $\mathrm{Hb}$ concentration (A), hematocrit (B), reticulocytes (C), and plasma EPO concentration (D) in normal rats treated with roxadustat three times per week for 29 days $(n=8$ per group). EPO concentration was measured 6 hours after the final dose. Data are presented as means \pm S.E.M. ${ }^{*} P<0.05$ vs. vehicle group. 

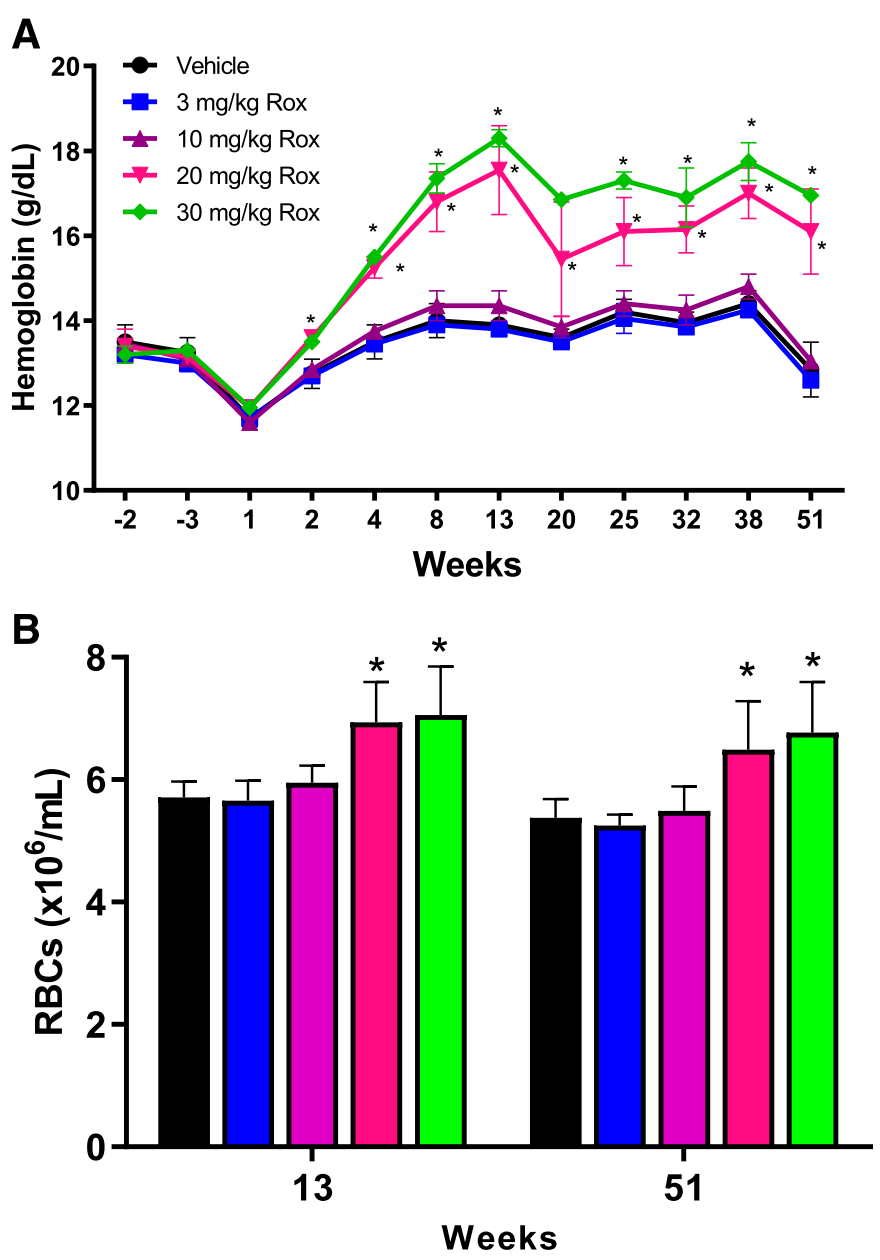

Fig. 5. Blood Hb concentration (A) and red blood cell (RBC) count (B) in cynomolgus monkeys treated with roxadustat three times per week for 52 weeks $(n=10$ per group). Data are presented as means \pm S.E.M. $* P<$ 0.05 vs. vehicle group.

including monocytes (Supplemental Fig. 4, A and B), neutrophils, lymphocytes, and eosinophils (data not shown). These animals also displayed increased paw weight, suggesting that they developed arthritis (Supplemental Fig. 4C). Treatment with roxadustat significantly decreased the monocyte count (Supplemental Fig. 4B) but did not affect other WBC types (data not shown). Darbepoetin and intravenous iron had no effect on the WBC response in PG-PS-challenged animals. Paw weight was not significantly affected by any of the treatment regimens (Supplemental Fig. 4C).

A multiplex cytokine panel was run on serum samples taken after 4 weeks of treatment. Values for IL- $1 \beta$, IL- 4 , TNF $\alpha$, and granulocyte-macrophage colony-stimulating factor were below the levels of quantitation in most samples (data not shown). IL-6 was primarily measurable in samples from PG-PS-challenged animals, and MIP-3a was measurable in nearly all samples. IL-6 and MIP-3a levels were not significantly reduced after treatment with roxadustat in PG-PS-challenged animals, although the data suggest a trending decline. Serum IL-6 was increased in the darbepoetin group, and intravenous iron had no effect on serum cytokines (Supplemental Fig. 4D).

Roxadustat Improves Iron Metabolism in a Rat Model of ACD. At the end of the ACD study, PG-PS-challenged animals treated with vehicle had lower serum iron (Fig. 8A) and transferrin saturation (Fig. 8B), as well as higher total iron-binding capacity (a measure of serum transferrin level, Fig. 8C), than vehicle-treated animals not challenged with PG-PS. In PG-PS-challenged animals, roxadustat significantly improved serum iron levels (Fig. 8A) and transferrin saturation (Fig. 8B) without affecting total iron-binding capacity (Fig. 8C) compared with vehicle. Neither darbepoetin nor intravenous iron had any significant effect on iron parameters compared with vehicle.

Quantitative PCR was used to quantify hepatic gene expression of Hamp (which encodes the protein hepcidin, a key regulator of iron metabolism and mediator of anemia of inflammation) and duodenal gene expression of Dcytb (a ferric reductase enzyme required for intestinal absorption of iron) and Dmt1 (a transporter of iron through apical membranes). Roxadustat reduced expression of hepatic Hamp (Fig. 9A) in PG-PS-challenged animals and increased expression of $D$ cyt $B$
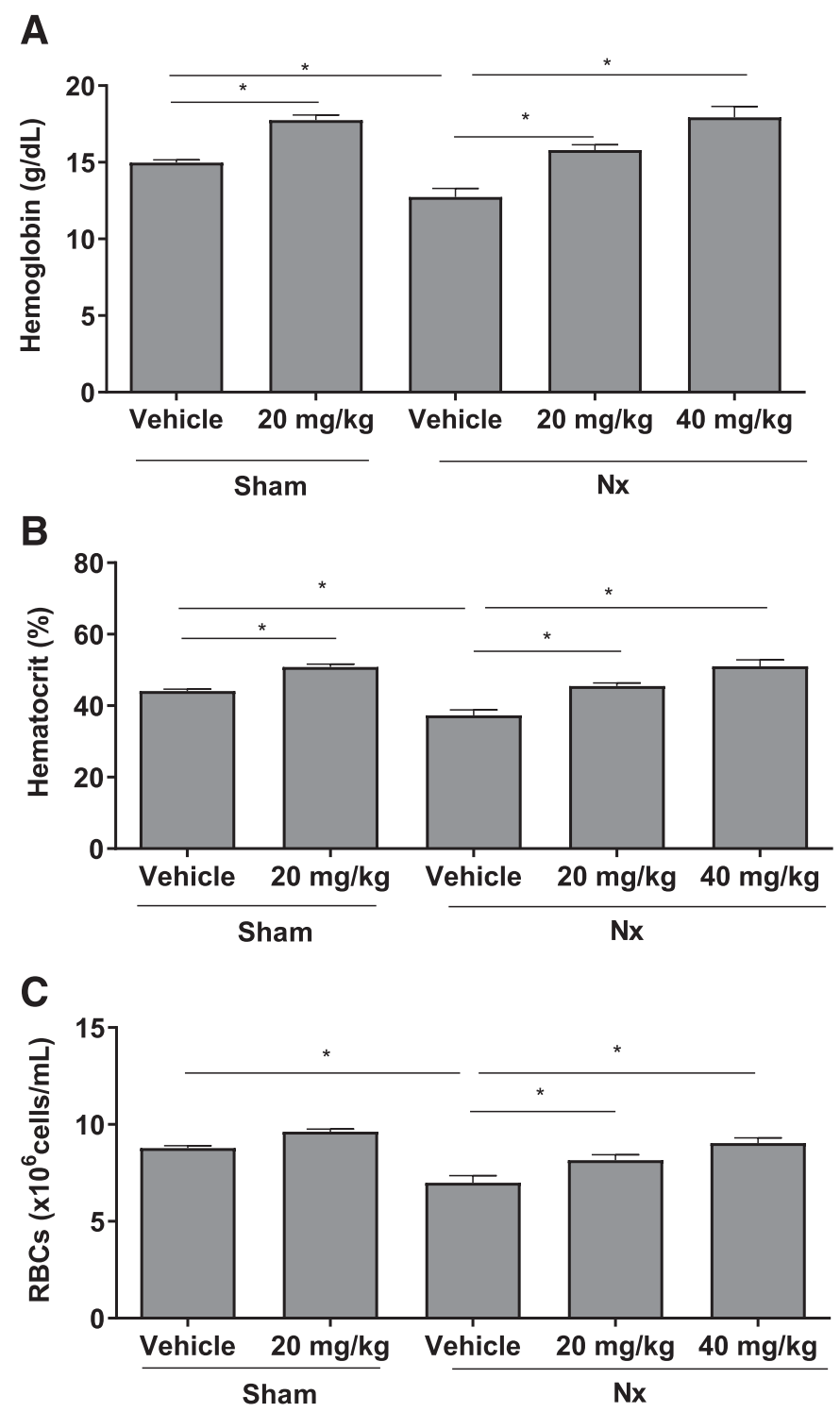

Fig. 6. Blood $\mathrm{Hb}$ concentration (A), hematocrit (B), and red blood cell (RBC) count (C) in rats after five-sixth nephrectomy (Nx) or sham surgery and treatment with roxadustat ( $n=8$ to 9 per group). Treatment (five doses over 2 weeks) was initiated 5 weeks after surgery. Data are presented as means \pm S.E.M. ${ }^{*} P<0.05$. 

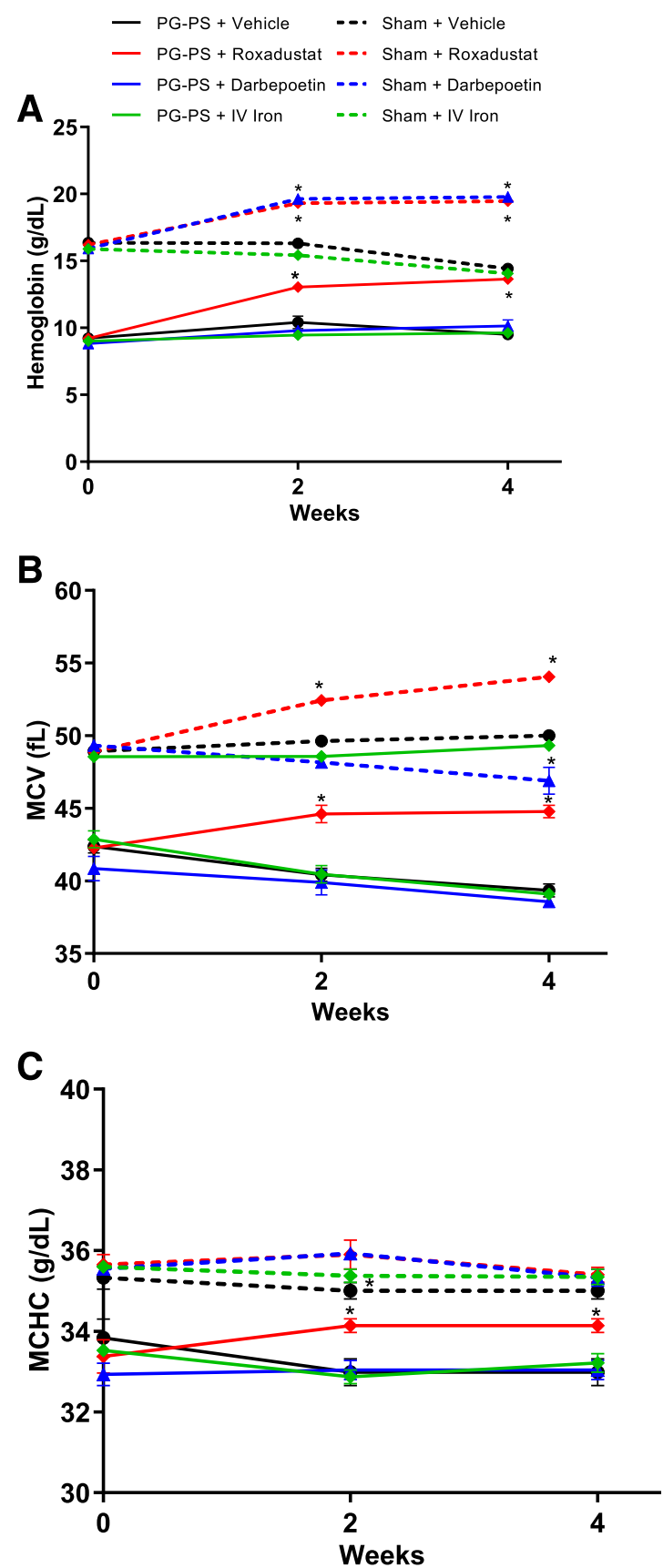

Fig. 7. Blood $\mathrm{Hb}$ concentration (A), $\mathrm{MCV}$ (B), and $\mathrm{MCHC}(\mathrm{C})$ in animals challenged with PG-PS ( $n=7$ to 8 ) and unchallenged (sham; $n=4$ ) animals treated with vehicle, $30 \mathrm{mg} / \mathrm{kg}$ roxadustat three times per week, darbepoetin alfa $(30 \mu \mathrm{g} / \mathrm{kg}$ every other week), or intravenous iron (IV iron; $1.5 \mathrm{mg} / \mathrm{kg}$ once per week). Treatment began 4 weeks after the PG-PS challenge and lasted for 4 weeks. Data are presented as means \pm S.E.M. $* P<0.05$ vs. corresponding (PG-PS-challenged or sham) vehicle group at the same time point.

(Fig. 9B) and Dmt1 (Fig. 9C) in the duodenum of PG-PS-challenged animals. Darbepoetin and intravenous iron had no significant effect on Hamp, DcytB, or Dmt1 expression.

\section{Discussion}

Several HIF-PH inhibitors are being evaluated in clinical trials as novel therapeutics to treat anemia of CKD (Gupta and Wish, 2017; Del Vecchio and Locatelli, 2018). In December 2018, roxadustat became the first HIF-PH inhibitor to receive marketing approval in any country when China's National Medical Products Administration approved it for the treatment of anemia of CKD in dialysis-dependent patients (Dhillon, 2019). We present here the pharmacological profile of roxadustat, an inhibitor of HIF-PH enzymes with similar potency for all three isoforms (PHD1, PHD2, and PHD3). The $\mathrm{IC}_{50}$ values for PHD1-3 were between 0.36 and $1.74 \mu \mathrm{M}$ under our assay conditions. Roxadustat displays competitive inhibition kinetics with respect to $\alpha \mathrm{KG}$, and therefore, the $\alpha \mathrm{KG}$ concentration in the enzyme reaction will significantly influence the calculated $\mathrm{IC}_{50}$. We chose $100 \mu \mathrm{M} \alpha \mathrm{KG}$ for our enzyme assays based on published reports of intracellular $\alpha \mathrm{KG}$ concentrations (Nagai et al., 1998; MacKenzie et al.,
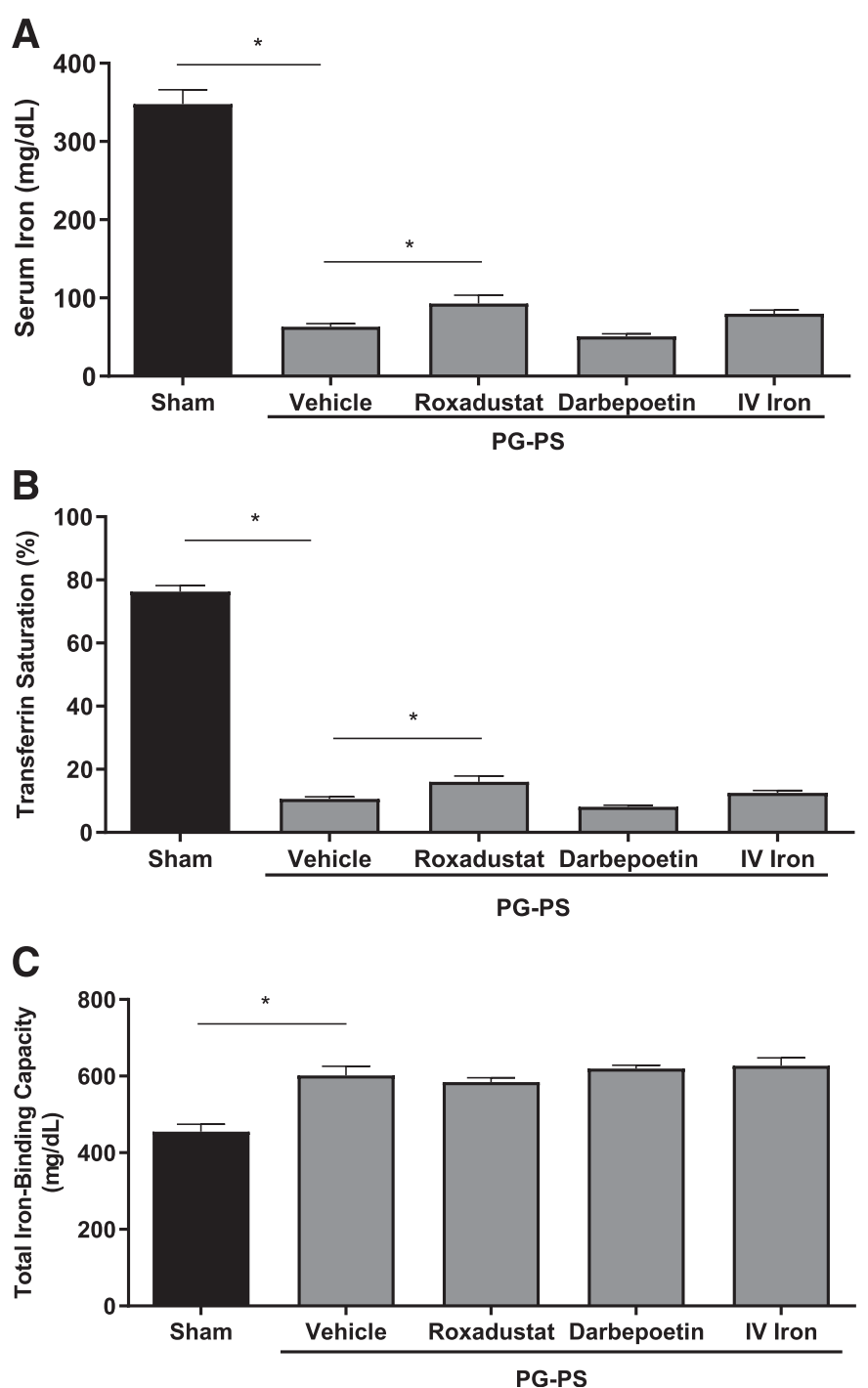

Fig. 8. Serum iron concentration (A), transferrin saturation $B$ ), and total iron-binding capacity (C) in PG-PS-challenged animals after 4 weeks of treatment with vehicle, $30 \mathrm{mg} / \mathrm{kg}$ roxadustat three times per week, darbepoetin alfa (30 $\mu \mathrm{g} / \mathrm{kg}$ every other week), or intravenous iron (IV iron; $1.5 \mathrm{mg} / \mathrm{kg}$ once per week) ( $n=7$ to 8$)$. All treatments were initiated 4 weeks after the PG-PS challenge. Sham (PG-PS-unchallenged) animals treated with vehicle are also plotted for comparison $(n=4)$. Data are presented as means \pm S.E.M. $* P<0.05$ vs. PG-PS vehicle group. 

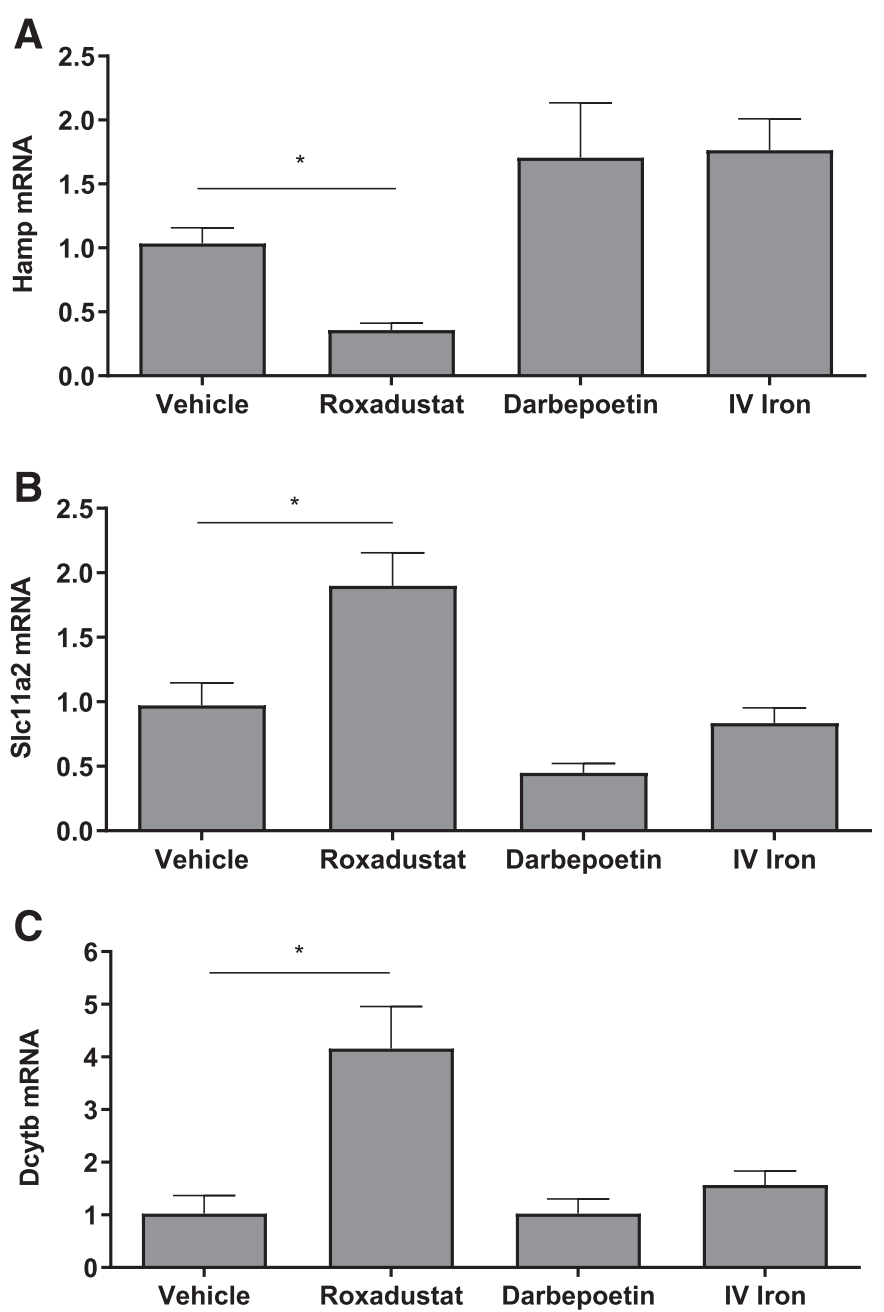

Fig. 9. mRNA expression of hepcidin (Hamp) in liver (A) and of iron transporter Dmt1 (Slc11a2) (B) and duodenal cytochrome b (Dcytb) (C) in duodenum of PG-PS-challenged animals after 4 weeks of treatment with vehicle, $30 \mathrm{mg} / \mathrm{kg}$ roxadustat three times per week, darbepoetin alfa (30 $\mu \mathrm{g} / \mathrm{kg}$ every other week), or intravenous iron (IV iron; $1.5 \mathrm{mg} / \mathrm{kg}$ once per week) $(n=6-8)$. All treatments were initiated 4 weeks after the PG-PS challenge. Gene expression normalized to $18 \mathrm{~S}$ ribosomal RNA is presented as fold-change vs. vehicle group, means \pm S.E.M. ${ }^{*} P<0.05$ vs. vehicle group.

2007). Assays carried out with lower $\alpha \mathrm{KG}$ concentrations would be expected to yield lower $\mathrm{IC}_{50}$ values (Yeh et al., 2017).

In cells, both HIF- $1 \alpha$ and HIF- $2 \alpha$ were stabilized by roxadustat at concentrations as low as $3 \mu \mathrm{M}$, consistent with the observation that roxadustat inhibits all three HIF-PH enzymes. HIF- $\alpha$ protein levels rapidly declined after washout of roxadustat from cell cultures, indicating that HIF-PH inhibition is reversible and that once the drug is removed, hydroxylation and degradation of HIF- $\alpha$ subunits quickly resumes.

Although HIF-1 was initially identified as the transcription factor responsible for the hypoxic induction of EPO (Semenza and Wang, 1992; Wang and Semenza, 1995; Wang et al., 1995), studies in mice and analysis of genetic mutations in patients with inherited polycythemia have since revealed that HIF-2 is the main regulator of EPO production (Rankin et al., 2007; Percy, 2008; Kapitsinou et al., 2010). In our studies, stabilization of HIF-2 $\alpha$ in Hep3B cells by roxadustat was accompanied by a dose-dependent increase in EPO production. HIF-2 also modulates intestinal iron uptake by regulating expression of DMT1 and DCYTB (Mastrogiannaki et al., 2009, 2012; Shah et al., 2009). However, other HIF-regulated genes involved in iron metabolism and erythropoiesis appear to depend more on HIF-1 than on HIF-2 (Rolfs et al., 1997; Tacchini et al., 1999; Zhang et al., 2011), suggesting that stabilization of both HIF-2 $\alpha$ and HIF- $1 \alpha$ is desirable for an optimal erythropoietic response.

Roxadustat exhibits considerable selectivity for the HIF-PH enzymes over the asparaginyl hydroxylase FIH, with an $\mathrm{IC}_{50}>150 \mu \mathrm{M}$ for FIH compared with $0.36-1.74 \mu \mathrm{M}$ for PHD1-3. Hydroxylation of HIF- $\alpha$ by FIH blocks the interaction between HIF- $\alpha$ and the p300/CBP family of coactivators and, as a result, limits the transcriptional activity of HIF (Mahon et al., 2001; Lando et al., 2002a,b). Certain HIF target genes are sensitive to FIH hydroxylation of HIF- $\alpha$ and are not optimally induced unless both HIF-PH enzymes and FIH are inhibited. Conversely, a subset of HIF target genes are relatively insensitive to FIH activity and can be induced by HIF-PH inhibition alone (Dayan et al., 2006; Chan et al., 2016). Because roxadustat inhibits HIF-PH enzymes but not FIH, the compound likely elicits a more limited HIF transcriptional response than conditions in which both HIF-PH and FIH are suppressed, such as severe hypoxia (Chan et al., 2016). The proangiogenic gene vascular endothelial growth factor (VEGF) is an example of an FIH-inhibited HIF target gene (Dayan et al., 2006). No increase in the risk of neoplasia was observed in nonclinical carcinogenicity studies and in VEGF-dependent tumor models (Beck et al., 2017; Seeley at al., 2017). Furthermore, changes in systemic VEGF levels with HIF-PH inhibitors such as roxadustat have not been reported in phase 2a studies (Locatelli et al., 2017).

Pharmacokinetic studies showed that roxadustat exhibited low clearance, high oral bioavailability, and half-lives of approximately 4 hours in rats and 11 hours in monkeys. Roxadustat administered orally three times per week to healthy rats for 29 days increased circulating EPO levels, blood $\mathrm{Hb}$, hematocrit, and reticulocytes in a dose-dependent manner. The increase in reticulocyte count was maximal 4-8 days after initiation of treatment. Blood $\mathrm{Hb}$ and hematocrit rose steadily over the 29 days of dosing. EPO plasma concentration peaked 6-10 hours after peak drug plasma level, consistent with upregulation of gene expression and protein synthesis. Plasma EPO levels remained elevated for a few hours and declined to baseline 24 hours after dosing, showing that transient stimulation of EPO production by intermittent dosing is sufficient for significant erythropoiesis to occur. Roxadustat was equally effective when administered intermittently to cynomolgus monkeys.

We studied the effect of roxadustat on anemia associated with chronic renal failure using five-sixth nephrectomized rats. This model is commonly used to study CKD and its complications, including anemia (de Mik et al., 2013; Garrido et al., 2015; Ribeiro et al., 2016). Roxadustat, administered for 2 weeks (20 and $40 \mathrm{mg} / \mathrm{kg}$ ) in nephrectomized rats, dosedependently normalized $\mathrm{Hb}$, hematocrit, and red blood cell count compared with control rats. In the clinical setting, roxadustat increased blood $\mathrm{Hb}$ in anemic $\mathrm{CKD}$ patients (Besarab et al., 2015, 2016; Provenzano et al., 2016a; Chen et al., 2019a,b).

Inflammatory cytokines such as TNF $\alpha$ and IL- $1 \beta$ suppress EPO production in cell culture and in isolated perfused rat 
kidneys (Jelkmann et al., 1994; La Ferla et al., 2002). This suppression may contribute to the pathophysiology of anemia of CKD. In Hep3B cells, we observed a decrease in EPO production after exposure to either TNF $\alpha$ or IL-1 $\beta$. Despite the suppression of basal EPO production by these inflammatory cytokines, roxadustat induced a dose-dependent increase in EPO in the presence of TNF $\alpha$ and IL-1 $\beta$.

The erythropoietic activity of roxadustat in the context of inflammation and impaired iron metabolism was evaluated in a rat model of anemia of inflammation induced by PG-PS. Four weeks of oral treatment with $30 \mathrm{mg} / \mathrm{kg}$ roxadustat three times per week corrected PG-PS-induced anemia in rats. Roxadustat also improved iron use, as shown by amelioration of microcytosis and hypochromia in PG-PS-injected animals. Serum iron and transferrin saturation were also mildly, but significantly, elevated, indicating improved mobilization of iron stores to support erythropoiesis. Hepcidin is a key regulator of iron metabolism and a mediator of anemia of inflammation (Deicher and Hörl, 2004). Roxadustat significantly decreased Hamp mRNA (hepcidin) expression in liver, which may explain the improved iron status and hematology observed in PG-PS-injected animals treated with roxadustat. Intestinal iron absorption by duodenal enterocytes requires DCYTB to reduce ferric iron to ferrous iron $\left(\mathrm{Fe}^{2+}\right)$ and DMT1 to transport $\mathrm{Fe}^{2+}$ through apical membranes. Roxadustat significantly increased $D c y t B$ and $D m t 1$ mRNA in the duodenum of PG-PS-injected animals, pointing to a potential mechanism by which iron availability is improved, consistent with HIF activation (Haase, 2010; Anderson et al., 2011). Roxadustat had little effect on inflammation in this model, indicating that the antianemic properties of roxadustat were not due to inhibition of the inflammatory response induced by PG-PS.

Conversely, the EPO analog darbepoetin administered every other week increased erythropoiesis in normal animals over a 4-week period but did not correct anemia of inflammation in the PG-PS model. The lack of efficacy of EPO analogs in this model was previously reported with recombinant human EPO (Barrett et al., 2011, 2015). These results indicate that darbepoetin is not as efficacious in stimulating erythropoiesis in the context of inflammation as in normal animals. Darbepoetin, unlike roxadustat, had no significant effect on iron metabolism, including serum iron, transferrin saturation, and mRNA expression of Hamp, DcytB, or Dmt1. These results may explain darbepoetin's lack of efficacy in this anemia model of functional iron deficiency (Sartor et al., 1989). Finally, the lack of effect of intravenous iron therapy in this study agrees with the functional iron deficiency seen in the PG-PS model. Iron supplementation alone could not overcome iron sequestration in these animals.

In clinical trials, roxadustat administered intermittently corrected anemia in CKD patients independently of iron or inflammatory status (Besarab et al., 2016; Provenzano et al., 2016a). It also reduced serum hepcidin levels and increased serum transferrin concentrations, improving iron delivery to bone marrow (Besarab et al., 2016; Chen et al., 2019a). Because roxadustat improved iron status, intravenous iron supplementation was not required for efficacy, in contrast to treatment with ESAs (Besarab et al., 2015; Provenzano et al., 2016a). Inflammation in patients with CKD is associated with hyporesponsiveness to ESAs, and the link between hyporesponsiveness and inflammation may be functional iron deficiency due to elevated hepcidin (MacDougall and Cooper, 2005; Adamson, 2009; Yilmaz et al., 2011). The coordinated erythropoiesis resulting from both increased EPO secretion and improved iron status explains the efficacy of roxadustat against anemia in the context of inflammation. It also accounts for the correction of anemia by roxadustat with relatively low concentrations of circulating endogenous EPO compared with the supraphysiologic serum EPO levels achieved after treatment with ESAs (Besarab et al., 2015; Provenzano et al., 2016b).

In conclusion, roxadustat inhibits all three HIF-PH isoforms, stabilizes HIF- $1 \alpha$ and HIF- $2 \alpha$, and dose-dependently increases EPO production in vitro, both in the absence and presence of inflammatory cytokines. Roxadustat exhibits erythropoietic activity in normal rats and monkeys and corrects anemia in rat models of CKD and of chronic inflammation with impaired iron metabolism. The coordinated erythropoietic response induced by roxadustat involving both increased EPO secretion and mobilization of iron stores makes this drug a promising treatment of anemia of CKD and anemia associated with functional iron deficiency.

\section{Acknowledgments}

The authors would like to thank Jenny Charan and Crystal Herron for editing the manuscript for submission. Roxadustat is being developed by FibroGen, Inc., AstraZeneca, and Astellas Pharma. The authors would like to thank Anja Slikkerveer (Astellas), Gerald Koelsh (Astellas), Tohru Ugawa (Astellas), Krister Bamberg (AstraZeneca), and Mike Rolf (AstraZeneca) for their support with the editing and reporting of this manuscript.

\section{Authorship Contributions}

Participated in research design: del Balzo, Signore, Walkinshaw, Seeley, Brenner, Wang, Guo, Flippin, Chow, Langsetmo, Guenzler, Liu, Klaus, Lin, Neff.

Conducted experiments: Wang, Guo, Arend, Chow, Gervasi, Kjaergaard, Langsetmo.

Performed data analysis: del Balzo, Signore, Walkinshaw, Seeley, Brenner, Wang, Guo, Chow, Gervasi, Langsetmo.

Wrote or contributed to the writing of the manuscript: del Balzo, Signore, Walkinshaw.

\section{References}

Adamson JW (2009) Hyporesponsiveness to erythropoiesis stimulating agents in chronic kidney disease: the many faces of inflammation. Adv Chronic Kidney Dis 16:76-82.

Anderson ER, Xue X, and Shah YM (2011) Intestinal hypoxia-inducible factor-2 $\alpha$ (HIF-2 $\alpha$ ) is critical for efficient erythropoiesis. J Biol Chem 286:19533-19540.

Babitt JL and Lin HY (2012) Mechanisms of anemia in CKD. J Am Soc Nephrol 23: 1631-1634.

Barrett TD, Palomino HL, Brondstetter TI, Kanelakis KC, Wu X, Haug PV, Yan W, Young A, Hua H, Hart JC, et al. (2011) Pharmacological characterization of 1-(5chloro-6-(trifluoromethoxy)- $1 H$-benzoimidazol-2-yl)-1H-pyrazole-4-carboxylic acid (JNJ-42041935), a potent and selective hypoxia-inducible factor prolyl hydroxylase inhibitor. Mol Pharmacol 79:910-920.

Barrett TD, Palomino HL, Brondstetter TI, Kanelakis KC, Wu X, Yan W, Merton KP Schoetens F, Ma JY, Skaptason J, et al. (2015) Prolyl hydroxylase inhibition corrects functional iron deficiency and inflammation-induced anaemia in rats. $\mathrm{Br}$ $J$ Pharmacol 172:4078-4088.

Beck J, Henschel C, Chou J, Lin A, and Del Balzo U (2017) Evaluation of the carcinogenic potential of roxadustat (FG-4592), a small molecule inhibitor of hypoxiainducible factor prolyl hydroxylase in CD-1 mice and Sprague Dawley rats. Int J Toxicol 36:427-439.

Besarab A, Chernyavskaya E, Motylev I, Shutov E, Kumbar LM, Gurevich K, Chan DTM, Leong R, Poole L, Zhong M, et al. (2016) Roxadustat (FG-4592): correction of anemia in incident dialysis patients. J Am Soc Nephrol 27:1225-1233.

Besarab A, Provenzano R, Hertel J, Zabaneh R, Klaus SJ, Lee T, Leong R, Hemmerich S, Yu K-HP, and Neff TB (2015) Randomized placebo-controlled doseranging and pharmacodynamics study of roxadustat (FG-4592) to treat anemia in nondialysis-dependent chronic kidney disease (NDD-CKD) patients. Nephrol Dial Transplant 30:1665-1673.

Biggar P and Kim G-H (2017) Treatment of renal anemia: erythropoiesis stimulating agents and beyond. Kidney Res Clin Pract 36:209-223.

Bunn HF (2013) Erythropoietin. Cold Spring Harb Perspect Med 3:a011619 DOI: 10.1101/cshperspect.a011619. 
Chan MC, Ilott NE, Schödel J, Sims D, Tumber A, Lippl K, Mole DR, Pugh CW, Ratcliffe PJ, Ponting CP, et al. (2016) Tuning the transcriptional response to hypoxia by inhibiting hypoxia-inducible factor (HIF) prolyl and asparaginyl hydroxylases. J Biol Chem 291:20661-20673.

Chen N, Hao C, Liu B-C, Lin H, Wang C, Xing C, Liang X, Jiang G, Liu Z, Li X, et al. (2019a) Roxadustat treatment for anemia in patients undergoing long-term dialysis. $N$ Engl J Med 381:1011-1022.

Chen N, Hao C, Peng X, Lin H, Yin A, Hao L, Tao Y, Liang X, Liu Z, Xing C, et al. (2019b) Roxadustat for anemia in patients with kidney disease not receiving dialysis. N Engl J Med 381:1001-1010.

Dayan F, Roux D, Brahimi-Horn MC, Pouyssegur J, and Mazure NM (2006) The oxygen sensor factor-inhibiting hypoxia-inducible factor-1 controls expression of distinct genes through the bifunctional transcriptional character of hypoxiainducible factor-1alpha. Cancer Res 66:3688-3698.

Deicher R and Hörl WH (2004) Hepcidin: a molecular link between inflammation and anaemia. Nephrol Dial Transplant 19:521-524.

Del Vecchio L and Locatelli F (2018) Investigational hypoxia-inducible factor prolyl hydroxylase inhibitors (HIF-PHI) for the treatment of anemia associated with chronic kidney disease. Expert Opin Investig Drugs 27:613-621.

de Mik SML, Hoogduijn MJ, de Bruin RW, and Dor FJMF (2013) Pathophysiology and treatment of focal segmental glomerulosclerosis: the role of animal models. BMC Nephrol 14:74

Dhillon S (2019) Roxadustat: first global approval. Drugs 79:563-572.

Finkelstein FO, Story K, Firanek C, Mendelssohn D, Barre P, Takano T, Soroka S, and Mujais S (2009) Health-related quality of life and hemoglobin levels in chronic kidney disease patients. Clin J Am Soc Nephrol 4:33-38.

Fong G-H and Takeda K (2008) Role and regulation of prolyl hydroxylase domain proteins. Cell Death Differ 15:635-641.

Garrido P, Ribeiro S, Fernandes J, Vala H, Bronze-da-Rocha E, Rocha-Pereira P, Belo L, Costa E, Santos-Silva A, and Reis F (2015) Iron-hepcidin dysmetabolism, anemia and renal hypoxia, inflammation and fibrosis in the remnant kidney rat model. PLoS One 10:e0124048 DOI: 10.1371/journal.pone.0124048.

Gupta N and Wish JB (2017) Hypoxia-inducible factor prolyl hydroxylase inhibitors: a potential new treatment for anemia in patients with CKD. Am J Kidney Dis 69 $815-826$.

Haase VH (2010) Hypoxic regulation of erythropoiesis and iron metabolism. Am J Physiol Renal Physiol 299:F1-F13.

Hirsilä M, Koivunen P, Xu L, Seeley T, Kivirikko KI, and Myllyharju J (2005) Effect of desferrioxamine and metals on the hydroxylases in the oxygen sensing pathway. FASEB J 19:1308-1310

Jelkmann WE, Fandrey J, Frede S, and Pagel H (1994) Inhibition of erythropoietin production by cytokines. Implications for the anemia involved in inflammatory states. Ann N Y Acad Sci 718:300-309, discussion 309-311.

Kaelin WG (2005) Proline hydroxylation and gene expression. Annu Rev Biochem 74: 115-128.

Kaelin WG Jr. and Ratcliffe PJ (2008) Oxygen sensing by metazoans: the central role of the HIF hydroxylase pathway. Mol Cell 30:393-402.

Kapitsinou PP, Liu Q, Unger TL, Rha J, Davidoff O, Keith B, Epstein JA, Moores SL, Erickson-Miller CL, and Haase VH (2010) Hepatic HIF-2 regulates erythropoietic responses to hypoxia in renal anemia. Blood 116:3039-3048.

Kassebaum NJ, Jasrasaria R, Naghavi M, Wulf SK, Johns N, Lozano R, Regan M, Weatherall D, Chou DP, Eisele TP, et al. (2014) A systematic analysis of global anemia burden from 1990 to 2010. Blood 123:615-624.

La Ferla K, Reimann C, Jelkmann W, and Hellwig-Bürgel T (2002) Inhibition of erythropoietin gene expression signaling involves the transcription factors GATA-2 and NF-kappaB. FASEB J 16:1811-1813.

Lando D, Peet DJ, Gorman JJ, Whelan DA, Whitelaw ML, and Bruick RK (2002a) FIH-1 is an asparaginyl hydroxylase enzyme that regulates the transcriptional activity of hypoxia-inducible factor. Genes Dev 16:1466-1471.

Lando D, Peet DJ, Whelan DA, Gorman JJ, and Whitelaw ML (2002b) Asparagine hydroxylation of the HIF transactivation domain a hypoxic switch. Science $\mathbf{2 9 5}$ 858-861.

Locatelli F, Fishbane S, Block GA, and Macdougall IC (2017) Targeting hypoxiainducible factors for the treatment of anemia in chronic kidney disease patients. Am J Nephrol 45:187-199.

Macdougall IC and Cooper AC (2005) Hyporesponsiveness to erythropoietic therapy due to chronic inflammation. Eur J Clin Invest 35 (Suppl 3):32-35.

MacKenzie ED, Selak MA, Tennant DA, Payne LJ, Crosby S, Frederiksen CM, Watson DG, and Gottlieb E (2007) Cell-permeating alpha-ketoglutarate derivatives alleviate pseudohypoxia in succinate dehydrogenase-deficient cells. Mol Cell Biol 27:3282-3289.

Mahon PC, Hirota K, and Semenza GL (2001) FIH-1: a novel protein that interacts with HIF-1alpha and VHL to mediate repression of HIF-1 transcriptional activity. Genes Dev 15:2675-2686.

Mastrogiannaki M, Matak P, Delga S, Deschemin J-C, Vaulont S, and Peyssonnaux C (2012) Deletion of HIF- $2 \alpha$ in the enterocytes decreases the severity of tissue iron loading in hepcidin knockout mice. Blood 119:587-590.

Mastrogiannaki M, Matak P, Keith B, Simon MC, Vaulont S, and Peyssonnaux C (2009) HIF-2alpha, but not HIF-1alpha, promotes iron absorption in mice. J Clin Invest 119:1159-1166.

Myllyharju J (2013) Prolyl 4-hydroxylases, master regulators of the hypoxia response. Acta Physiol (Oxf) 208:148-165.

Naets JP (1963) Relation between erythropoietin plasma level and oxygen requirements. Proc Soc Exp Biol Med 112:832-836.
Nagai J, Yano I, Hashimoto Y, Takano M, and Inui K (1998) Efflux of intracellular alpha-ketoglutarate via p-aminohippurate/dicarboxylate exchange in OK kidney epithelial cells. J Pharmacol Exp Ther 285:422-427.

Percy MJ (2008) Familial erythrocytosis arising from a gain-of-function mutation in the HIF2A gene of the oxygen sensing pathway. Ulster Med $J$ 77:86-88.

Peyssonnaux C, Nizet V, and Johnson RS (2008) Role of the hypoxia inducible factors HIF in iron metabolism. Cell Cycle 7:28-32.

Pfeffer MA, Burdmann EA, Chen CY, Cooper ME, de Zeeuw D, Eckardt KU, Feyzi JM, Ivanovich P, Kewalramani R, Levey AS, et al.; TREAT Investigators (2009) A trial of darbepoetin alfa in type 2 diabetes and chronic kidney disease. $N$ Engl J Med 361:2019-2032.

Prentice TC and Mirand EA (1961) Effect of hypoxia on plasma erythropoietin in the rabbit. Proc Soc Exp Biol Med 106:501-502.

Provenzano R, Besarab A, Sun CH, Diamond SA, Durham JH, Cangiano JL, Aiello JR, Novak JE, Lee T, Leong R, et al. (2016a) Oral hypoxia-inducible factor prolyl hydroxylase inhibitor roxadustat (FG-4592) for the treatment of anemia in patients with CKD. Clin J Am Soc Nephrol 11:982-991.

Provenzano R, Besarab A, Wright S, Dua S, Zeig S, Nguyen P, Poole L, Saikali KG, Saha G, Hemmerich S, et al. (2016b) Roxadustat (FG-4592) versus epoetin alfa for anemia in patients receiving maintenance hemodialysis: a phase 2 , randomized, 6 to 19-week, open-label, active-comparator, dose-ranging, safety and exploratory efficacy study. Am J Kidney Dis 67:912-924.

Rankin EB, Biju MP, Liu Q, Unger TL, Rha J, Johnson RS, Simon MC, Keith B, and Haase VH (2007) Hypoxia-inducible factor-2 (HIF-2) regulates hepatic erythropoietin in vivo. $J$ Clin Invest 117:1068-1077.

Ribeiro S, Garrido P, Fernandes J, Vala H, Rocha-Pereira P, Costa E, Belo L, Reis F, and Santos-Silva A (2016) Pathological and molecular mechanisms underlying resistance to recombinant human erythropoietin therapy in the remnant kidney rat model of chronic kidney disease associated anemia. Biochimie 125:150-162.

Rolfs A, Kvietikova I, Gassmann M, and Wenger RH (1997) Oxygen-regulated transferrin expression is mediated by hypoxia-inducible factor-1. J Biol Chem 272: 20055-20062.

Sartor RB, Anderle SK, Rifai N, Goo DA, Cromartie WJ, and Schwab JH (1989) Protracted anemia associated with chronic, relapsing systemic inflammation induced by arthropathic peptidoglycan-polysaccharide polymers in rats. Infect Immun 57:1177-1185.

Seeley TW, Sternlicht MD, Klaus SJ, Neff TB, and Liu DY (2017) Induction of erythropoiesis by hypoxia-inducible factor prolyl hydroxylase inhibitors without promotion of tumor initiation, progression, or metastasis in a VEGF-sensitive model of spontaneous breast cancer. Hypoxia (Auckl) 5:1-9.

Semenza GL and Wang GL (1992) A nuclear factor induced by hypoxia via de novo protein synthesis binds to the human erythropoietin gene enhancer at a site required for transcriptional activation. Mol Cell Biol 12:5447-5454.

Shah YM, Matsubara T, Ito S, Yim S-H, and Gonzalez FJ (2009) Intestinal hypoxiainducible transcription factors are essential for iron absorption following iron deficiency. Cell Metab 9:152-164.

Stauffer ME and Fan T (2014) Prevalence of anemia in chronic kidney disease in the United States. PLoS One 9:e84943 DOI: 10.1371/journal.pone.0084943.

Szczech LA, Barnhart HX, Inrig JK, Reddan DN, Sapp S, Califf RM, Patel UD, and Singh AK (2008) Secondary analysis of the CHOIR trial epoetin- $\alpha$ dose and achieved hemoglobin outcomes. Kidney Int 74:791-798.

Tacchini L, Bianchi L, Bernelli-Zazzera A, and Cairo G (1999) Transferrin receptor induction by hypoxia. HIF-1-mediated transcriptional activation and cell-specific post-transcriptional regulation. J Biol Chem 274:24142-24146.

Thomas R, Kanso A, and Sedor JR (2008) Chronic kidney disease and its complications. Prim Care 35:329-344, vii.

Thorp ML, Johnson ES, Yang X, Petrik AF, Platt R, and Smith DH (2009) Effect of anaemia on mortality, cardiovascular hospitalizations and end-stage renal disease among patients with chronic kidney disease. Nephrology (Carlton) 14:240-246.

Tian H, McKnight SL, and Russell DW (1997) Endothelial PAS domain protein 1 (EPAS1), a transcription factor selectively expressed in endothelial cells. Genes Dev 11:72-82.

Wang GL, Jiang BH, Rue EA, and Semenza GL (1995) Hypoxia-inducible factor 1 is a basic-helix-loop-helix-PAS heterodimer regulated by cellular O2 tension. Proc Natl Acad Sci USA 92:5510-5514.

Wang GL and Semenza GL (1995) Purification and characterization of hypoxiainducible factor 1. J Biol Chem 270:1230-1237.

Yeh T-L, Leissing TM, Abboud MI, Thinnes CC, Atasoylu O, Holt-Martyn JP, Zhang D, Tumber A, Lippl K, Lohans CT, et al. (2017) Molecular and cellular mechanisms of HIF prolyl hydroxylase inhibitors in clinical trials. Chem Sci (Camb) 8: $7651-7668$

Yilmaz MI, Solak Y, Covic A, Goldsmith D, and Kanbay M (2011) Renal anemia of inflammation: the name is self-explanatory. Blood Purif 32:220-225.

Zhang F-L, Shen G-M, Liu X-L, Wang F, Zhao H-L, Yu J, and Zhang J-W (2011) Hypoxic induction of human erythroid-specific $\delta$-aminolevulinate synthase mediated by hypoxia-inducible factor 1. Biochemistry 50:1194-1202.

Zhang JH, Qi RC, Chen T, Chung TD, Stern AM, Hollis GF, Copeland RA, and Oldenburg KR (1999) Development of a carbon dioxide-capture assay in microtiter plate for aspartyl-beta-hydroxylase. Anal Biochem 271:137-142.

Address correspondence to: Ughetta del Balzo, FibroGen, Inc., 409 Illinois Street, San Francisco, CA 94158. E-mail: udelbalzo@fibrogen.com 